Intersections

Canadian Journal of Music

Revue canadienne de musique
Intersections CANADIAN JOURAL OF MUSIC
REVUE CANADIENEE DE MUSIOUH

\title{
Quels sont les paradigmes didactiques présents dans les méthodes de guitare destinées aux débutants : expérimentation d'une méthodologie d'analyse
}

\section{Isabelle Héroux}

Volume 35, numéro 2, 2015

URI : https://id.erudit.org/iderudit/1043821ar

DOI : https://doi.org/10.7202/1043821ar

Aller au sommaire du numéro

\section{Éditeur(s)}

Canadian University Music Society / Société de musique des universités canadiennes

ISSN

1911-0146 (imprimé)

1918-512X (numérique)

Découvrir la revue

Citer cet article

Héroux, I. (2015). Quels sont les paradigmes didactiques présents dans les méthodes de guitare destinées aux débutants : expérimentation d'une méthodologie d'analyse. Intersections, 35(2), 37-74.

https://doi.org/10.7202/1043821ar
Résumé de l'article

Cet article présente une analyse de méthodes de guitare classique pour débutants puisées dans les programmes d'études pour l'enseignement privé reconnu par le gouvernement du Québec. Pour identifier les principes didactiques sous-jacents à la conception des ouvrages, nous avons analysé les éléments d'apprentissages proposés, leur ordre de présentation ainsi que le type et la quantité d'activités pour chacun. L'apprentissage des notes, de leur lecture et des techniques de jeu dans le but de reproduire le texte musical à l'instrument constitue l'essentiel de ce qui est présenté dans ces méthodes, dans des proportions variables souvent en lien avec l'âge ciblé.
Copyright @ Canadian University Music Society / Société de musique des universités canadiennes, 2018
Ce document est protégé par la loi sur le droit d'auteur. L'utilisation des services d'Érudit (y compris la reproduction) est assujettie à sa politique d'utilisation que vous pouvez consulter en ligne. 


\title{
QUELS SONT LES PARADIGMES DIDACTIQUES PRÉSENTS DANS LES MÉTHODES DE GUITARE DESTINÉES AUX DÉBUTANTS : EXPÉRIMENTATION D'UNE MÉTHODOLOGIE D'ANALYSE
}

\author{
Isabelle Héroux
}

\section{Contexte}

Dans plusieurs disciplines artistiques, l'enseignement s'effectue par un maître qui assure la transmission d'un savoir à un élève qui devient à son tour héritier d'une tradition (Bureau \& Burns 1990, p. 22). En musique, on retrouve des traces de ce type d'enseignement individualisé dès l'Antiquité, grâce à des hiéroglyphes, à des gravures et à d'autres témoignages (Favre 1988, p. 15). Tout comme l'enseignement par compagnonnage chez les artisans, cette tradition repose sur la transmission orale des connaissances du maître à son apprenti, et ce, jusqu'à la première moitié du XVIII siècle (Lescat 1991, p. 134). Cependant, avec le développement des instruments de musique et des techniques qui leur sont propres, les défis que pose l'acquisition d'habiletés psychomotrices toujours plus complexes rendent l'apprentissage instrumental difficile (Lartigot 1999, p.14). Dès lors, on voit apparaître une formalisation de l'enseignement qui se traduit par l'apparition de méthodes sous forme de livres imprimés, destinés à l'apprentissage d'instruments spécifiques ${ }^{1}$ ainsi que par l'avènement d'institutions d'enseignement de la musique (pensons notamment à l'Institut national de la musique fondé en 1795, ancêtre des conservatoires). Ainsi, dès la fin du XVIII ${ }^{\mathrm{e}}$ siècle, on voit émerger une pédagogie instrumentale, ce que Gauthier appelle le «savoir enseigner» (2012, p. 63), qui propose «la reproduction des savoir-faire suivant une progression typée, une articulation des difficultés à dominer dans une durée donnée, des répertoires d'application construits pour la circonstance ...»

De nos jours, l'enseignement d'un instrument de musique est souvent effectué en dyade enseignant et élève, comme nous pouvons le constater dans l'offre de cours des écoles préparatoires de musique de l'UQAM ou du Collègue

1 Auparavant, les méthodes étaient destinées à l'apprentissage du chant, avec en annexe, des instructions spécifiques pour l'apprentissage des instruments. Le clavecin est le premier instrument pour lequel des méthodes furent écrites (Lartigot 1999, p. 13). 
Vincent d'Indy ${ }^{2}$. On voit certes l'enseignement instrumental en groupe dans les écoles primaires et secondaires; cependant, que ce soit pour le loisir ou pour la formation de futurs musiciens professionnels dans les CÉGEPS, universités et conservatoires, les leçons individuelles consacrées à l'apprentissage d'un répertoire musical propre à l'instrument choisi par l'étudiant sont toujours présentes. Il appert que cet enseignement repose encore de nos jours sur la tradition et la transmission de connaissances informelles acquises au fil des siècles (Comeau 2006, p. 3). Ainsi, le musicien transmet à son élève le savoir-faire qu'il a reçu de son maître (dont il reproduit souvent le type d'enseignement) et utilise le matériel qui a servi à son apprentissage ou, encore, en conçoit qui s'appuie sur les mêmes pratiques, sans qu'il y ait renouvellement véritable de celles-ci (Héroux 2006, p. 4). Ainsi, il est pertinent de penser qu'un ensemble de croyances, de règles et de valeurs agissent comme cadre de référence et conditionnent les choix pédagogiques des musiciens enseignants selon la tradition dont ces derniers sont issus. L'identification de ces principes pédagogiques pourrait permettre une réflexion approfondie sur la pédagogie instrumentale ainsi qu'une remise en question de pratiques traditionnelles à la lumière des récentes recherches. Une question vient alors à l'esprit: comment identifier de tels paradigmes?

Comme l'enseignement instrumental est soutenu par du matériel éducatif qui reflète, du moins en partie, les convictions pédagogiques du musicien pédagogue et la tradition dont il est issu, ce matériel pourrait nous permettre d'identifier les principes qui guident l'enseignement de la guitare classique offert aux débutants. En effet, en identifiant les éléments enseignés (notes, techniques de jeu), les manières dont ils sont présentés aux apprentis guitaristes (pièces, exercices) et dans quel ordre ils apparaissent, nous croyons possible d'identifier certains paradigmes qui guident l'enseignement de la guitare classique aux débutants de la province de Québec. Cet article présente donc les résultats d'une analyse de matériel éducatif pour débutants utilisé dans l'enseignement de la guitare classique au Québec.

\section{REVUE DE LITTÉRATURE}

L’analyse du matériel éducatif est plus courante dans le domaine de l'enseignement du piano, quels que soient l'âge et le niveau. Lu (2012, p. 22) note que les études qui s'intéressent au matériel éducatif pour piano se concentrent soit sur l'analyse du répertoire musical, soit sur la progression des apprentissages. De plus, si certaines reposent sur une méthodologie scientifique, elles sont également souvent réalisées par les pédagogues eux-mêmes et publiées dans des revues professionnelles.

Lu (2012) analyse de manière approfondie le répertoire présent dans 18 séries de Method Book, qu'elle définit comme des séries de livres comprenant des leçons et du matériel additionnel écrit dans le but d'offrir un programme

2 Vous pouvez consulter les sites internet de www.epm.uqam.ca et http://www.emvi.qc.ca/ pour plus de détails sur les cours offerts. 
complet d'apprentissage du piano en s'inspirant de la définition d'Albergo (1988) ${ }^{3}$. Ces ouvrages, tels les Bastien Piano Basics (Bastien 1985) et Piano Adventures (Faber \& Faber 1996), ont été édités entre 1981 et 2010; ils proviennent d'Amérique du Nord et comprennent entre quatre et neuf volumes. Selon cette étude, le répertoire écrit par les auteurs représente $60 \%$ de ce qui est présenté dans les méthodes pour piano, souvent dans des pièces qui imitent différents styles musicaux, par exemple la musique romantique ou le jazz. La musique traditionnelle représente $18 \%$ du répertoire comptabilisé dans les ouvrages, le tout complété par du répertoire de musique classique et populaire que $\mathrm{Lu}$ détaille en différentes sous-catégories. Elle note une seule pièce de la Renaissance dans tout son corpus (p. 76). Cette analyse de 18 séries de méthodes se concentre sur un seul élément, le type de répertoire présenté. Le but avoué est simple: aider les professeurs de piano à choisir les méthodes les plus adaptées à leurs besoins et à ceux des élèves (p. 94). Ainsi, l'auteure n'a pas relevé les valeurs pédagogiques qui expliquent le choix des auteurs d'inclure ou non un certain type de répertoire.

Sundell (2012, p. 42) quant à elle s'intéresse au contenu des tout premiers niveaux offerts dans les volumes de 12 séries de Method Books destinés aux pianistes débutants de six ans et plus, éditées entre 1950 et 2003 (entre autres Bastien Piano Basics et Piano Adventures). Contrairement à Lu (2012), elle ne s'intéresse pas au jeu instrumental à proprement parler, mais plutôt à la présence d'éléments en lien avec le comprehensive musicianship ${ }^{4}$ qu'elle définit comme l'intégration d'éléments autres que la technique instrumentale ou le travail du répertoire musical. Ces éléments pourraient être, par exemple, des activités liées à la théorie musicale, au développement de la perception auditive, à l'histoire, à la composition ou à l'improvisation. Dans cette étude, l'auteure va plus loin que le dénombrement des activités proposées dans les méthodes: elle analyse aussi les buts pédagogiques visés par les activités. Si l'auteure constate peu d'activités d'apprentissage liées à l'histoire de la musique, à la composition, à la transposition ou à l'improvisation dans le matériel analysé, elle note que celles liées à la théorie musicale sont majoritaires. Il va de soi, selon Sundell (2012), que le besoin de développer les habiletés de lecture à vue et la compréhension du texte musical justifient cette présence. Les activités liées à la perception auditive sont quant à elles bien présentes. Elles prennent souvent la forme de musique d'ensemble, composées principalement de duos dans lesquels l'enseignant accompagne l'élève. L'auteur attribue la cause de cette représentation à l'aspect motivationnel que ce type d'activités apporte à l'apprenant (p. 70). Les résultats de cette étude nous permettent de constater que le travail de la technique instrumentale et du répertoire est l'ultime but visé dans les méthodes analysées. Ainsi, assurer le développement musical général ne semble pas être une valeur importante. De plus, le fait que l'auteur constate qu'il n'y a pas eu de variation significative dans la représentativité des catégories liées

3 «A series of lesson books and supplementary materials written to create a planned program of piano study.» d'Albergo 1988, p. 22.

4 Le terme n'a pas d'équivalent en français, mais il peut être traduit par l'approche globale de la musique. 
au comprehensive musicianship selon les années de publication des ouvrages étudiés (p. 6o) est surprenant.

Rares sont les recherches consacrées au matériel éducatif pour la guitare classique. Ainsi, un seul article grand public a été recensé, "Un éventail des méthodes de guitare» de Delcamp (1984), qui présente 24 ouvrages du XIX ${ }^{\mathrm{e}}$ et $\mathrm{du} \mathrm{XX}^{\mathrm{e}}$ siècles destinés à l'enseignement de la guitare, dans la revue Les Cahiers de la guitare, $\mathrm{n}^{\circ} 12$, 1984. Par contre, l'auteur propose davantage un inventaire des ouvrages disponibles sur le marché selon les âges qu'une analyse critique des valeurs pédagogiques qui ont participé à leur création. Du côté des recherches académiques, si Valois (2009) et Ribouillot (1980) ont analysé des méthodes de guitare ainsi que des partitions publiées en France aux XVIIIe et $\mathrm{XIX}^{\mathrm{e}}$ siècles, ils visaient cependant à comprendre l'évolution des techniques de jeu utilisées à cette époque d'un point de vue musicologique. Héroux (2006) adopte une posture pédagogique dans son analyse du matériel éducatif destiné aux étudiants guitaristes qui ne sont pas débutants. En effet, le public ciblé est de niveau intermédiaire - inscrit au programme collégial de musique -, soit dans une formation professionnelle préuniversitaire. Lauteure analyse le contenu de différents ouvrages disponibles sur le marché, en lien avec les besoins de formation identifiés dans le programme de musique développé par le gouvernement québécois. Le but ici n'est pas d'identifier les paradigmes pédagogiques présents, mais bien de déterminer les lacunes de cette littérature spécialisée afin de proposer un nouvel ouvrage adapté à l'enseignement préuniversitaire de la guitare.

Ainsi, à notre connaissance, seule Sodoyer (1995) a effectué une analyse du matériel éducatif dont l'objet était de favoriser l'apprentissage de la guitare auprès des débutants au $\mathrm{XX}^{\mathrm{e}}$ siècle. Plus précisément, elle s'est intéressée auX méthodes utilisées en France par des professeurs et a analysé 49 ouvrages publiés entre 1901 et 1994, pour ainsi tracer les grandes lignes de l'évolution de leur contenu. L'intérêt de cette analyse tient au fait que la chercheure prend en compte plusieurs éléments présents dans les méthodes (la provenance du répertoire, les activités liées au comprehensive musicianship), mais s'attarde également aux types d'exercices techniques proposés et à la manière dont les auteurs présentent l'apprentissage des notes. Elle constate différents changements dans les méthodes publiées à partir de 1970 (Ibid., p. 106-110). Ainsi, avant 1970, les notes devaient toutes être apprises au même moment dans des gammes complètes qui structuraient souvent les chapitres des méthodes. À partir de 1970, les notes sont introduites de manière plus graduelle sans répondre à une logique de gammes. Un autre changement à noter réside dans l'abandon des techniques de jeu complexes et exigeantes (barré, harmonique, coulé) au profit de techniques plus simples qui permettent de jouer un répertoire monodique et en duo, remplaçant dès lors les œuvres polyphoniques qui constituaient la majorité du répertoire alors proposé aux débutants.

Sodoyer (1995, p. 105-108) constate aussi que jusque dans les années 1970, la majorité des auteurs composaient l'entièreté du répertoire des méthodes, et que celui-ci était souvent accompagné de suggestions d'œuvres complémentaires à consulter. Cette tendance s'est cependant inversée en faveur de compilations 
d'œuvres de diverses provenances. Les pièces de la Renaissance apparaissent en 1963 dans les méthodes de guitare. Elles gagnent en popularité dans les années 1970, étant présentes à raison d'un à quatre morceaux dans les deux tiers des ouvrages. Le répertoire baroque apparaît quant à lui plus tôt, soit en 1955, pour être plus présent entre les années 1960 et 1975 dans trois ouvrages sur quatre. Par la suite, sa présence décroit. Le répertoire de la période classique, s'il est présent en 1956, est presque inexistant, ceci étant attribuable au fait que peu d'œuvres ont été composées pour guitare à cette époque. Par contre, la majorité du répertoire original pour la guitare provient de l'époque romantique, un âge d'or pour l'instrument. L'auteur constate que depuis les années 1960, 37 méthodes intègrent des œuvres romantiques.

La qualité du son est abordée dans 29 méthodes sur 49, principalement pour évoquer la possibilité de changer de timbre, mais rarement pour parler de la qualité sonore générale. Par exemple, les auteurs proposent de jouer avec la main droite près du chevalet pour obtenir un timbre plus métallique, alors que pour un timbre plus velouté, ils suggèrent de s'en éloigner. Quant à l'interprétation générale des œuvres, à l'expressivité ou au caractère, 17 méthodes n'y font pas allusion avant 1960, mais certaines y consacrent un chapitre distinct, en fin de manuel. Les nuances sont présentes dans presque tous les ouvrages analysés, mais avant 1970, on ne les voit apparaître qu'à la fin des méthodes, et ce, seulement dans quelques morceaux seulement (Ibid., p. 114). Par contre, après les années 1960, c'est tout au long des méthodes que l'on retrouve des éléments en lien avec l'expressivité. L'auteur constate donc qu'avant les années 1960, les méthodes valorisaient la maitrise de différentes techniques de jeu complexes avant d'aborder l'expressivité, alors que depuis les années 1970, les éléments techniques exigés sont plus simples et les éléments liés à l'expressivité sont introduits plus tôt (Ibid., p. 116).

Dans le corpus étudié par Sodoyer (1995, p. 118), les activités qui demandent d'improviser apparaissent en 1983 chez Membrado, avec un exercice dans lequel l'élève doit inventer des rythmes sur une mélodie de Bartók et les jouer en pizzicato. Par la suite, huit méthodes suggèrent des exercices d'improvisation mélodique avec plus ou moins de consignes sur les notes, rythmes et nuances à utiliser. Dès 1989, Sodoyer constate l'apparition de parties d'accompagnement dans les méthodes afin de rendre les exercices plus intéressants. En conclusion, Sodoyer (1995) attribue les changements constatés dans les méthodes de guitare au fait que la clientèle ciblée par celles-ci a évolué. En effet, les nouvelles méthodes s'adressent principalement aux enfants qui sont de plus en plus présents dans les écoles de musique. Ainsi, selon l'auteure, la conception des ouvrages change en fonction des clientèles visées, mais les conceptions pédagogiques qui soutiennent ces changements ne sont pas explicitement nommées.

Il est intéressant de constater que les ouvrages de Lu et Sundell sont complémentaires: l'un étudie le répertoire proposé dans les méthodes, alors que l'autre s'attarde à ce qui ne touche pas le jeu instrumental en tant que tel, soit le comprehensive musicianship. Comme Sodoyer étudie l'évolution du contenu des méthodes, elle s'intéresse à tout ce qui est présent, du répertoire aux éléments du comprehensive musicianship, en passant par la technique instrumentale. 
La revue de littérature met en évidence le peu d'étude sur le matériel éducatif utilisé pour l'enseignement instrumental aux débutants. De plus, les recherches consultées visent plutôt à identifier ce qui est présent dans le matériel éducatif qu'à comprendre les valeurs pédagogiques qui pourraient expliquer leur présence ou leur absence. Si les méthodologies employées dans celles-ci permettent d'en identifier le contenu de manière pertinente et d'effectuer une comparaison entre les ouvrages analysés, elles ne visent pas une réflexion sur les aspects axiologiques présents dans les méthodes. Pourtant, un tel travail pourrait nous éclairer sur les paradigmes présents et permettre une réflexion approfondie sur les pratiques pédagogiques. Ainsi, nous proposons dans cet article l'expérimentation d'une méthodologie qui vise à alimenter une telle réflexion sur l'enseignement de la guitare classique aux débutants. Nous avons voulu identifier les éléments présents dans les méthodes, par exemple, l'apprentissage des notes et de leur lecture, des techniques de jeu, des nuances et autres. De plus, nous avons voulu savoir de quelles manières ils sont présentés aux apprentis guitaristes. Est-ce grâce à des explications, des exercices avec ou sans lecture de la partition, du répertoire? Finalement, nous avons voulu savoir dans quel ordre sont présentés ces différents éléments dans les méthodes. L’objectif de cette recherche était de développer une méthodologie permettant d'identifier les paradigmes qui guident le développement de méthodes imprimées pour l'enseignement de la guitare classique destinées aux débutants.

\section{MÉTHOdologie}

Les ouvrages consacrés à la guitare classique disponibles sur le marché sont nombreux, s'adressent à différentes clientèles (enfants, adolescents, adultes), de niveaux variés (débutant, intermédiaire ou avancé) et ils sont présentés sous différents formats (imprimés, numériques, avec support audio ou autres). La première étape de cette étude a permis de déterminer les ouvrages à analyser. La délimitation du corpus s'est faite selon les quatre règles d'analyse de contenu énoncées par Bardin (2003, p. 127), afin de s'assurer que les informations recueillies soient nécessaires, suffisantes et fidèles à la réalité. Ainsi, par souci d'homogénéité du corpus, nous avons retenu le matériel éducatif dont l'objet était l'apprentissage de la guitare classique aux débutants de o à 2 années d'expérience. Nous avons aussi conservé uniquement les ouvrages qui sont des méthodes selon la définition de Costenza et Russell (1992), soit des publications qui traitent de plus d'un sujet (par exemple, des exercices techniques et du répertoire) avec une mise en forme pédagogique et un ordre de présentation séquentiel du contenu dans le but d'en faciliter l'apprentissage, en opposition avec un outil, qui aborde un seul sujet (comme un livre ne contenant que des gammes ou seulement du répertoire). Afin de nous assurer de la pertinence, de la représentativité et de l'exhaustivité du corpus, nous avons retenu les ouvrages qui sont recommandés dans les programmes d'études des organismes 
d'encadrement de l'enseignement privé de la musique reconnus par le Ministère de l'Éducation et de l'enseignement supérieur (MEES)5.

\section{Organismes d'encadrement et matériel éducatif}

Il existe onze organismes d'encadrement de l'enseignement privé de la musique qui proposent des programmes d'études pour l'apprentissage de différents instruments de musique ${ }^{6}$. Ces programmes d'études proposent un cheminement pédagogique gradué et organisé en niveaux. Les musiciens pédagogues peuvent s'affilier à ces organismes d'encadrement, profiter du soutien pédagogique qui y est offert et utiliser les programmes pour baliser leur enseignement en privé. Après une année d'études, selon les recommandations du programme, les élèves de l'enseignant affilié peuvent passer un examen qui est administré par l'organisme d'encadrement. Celui-ci décerne alors un certificat pour marquer la réussite du niveau. Ces programmes d'études sont assujettis à certaines normes et à des évaluations aux cinq ans par le MEES. Pour cette étude, nous avons évalué les programmes pour l'enseignement de la guitare qui étaient en vigueur de 2010 à 2015. Les organismes d'encadrement qui proposaient des programmes d'enseignement de la guitare étaient au nombre de huit, dont deux sont des organisations privées et six sont rattachées à des universités. En voici la liste:

Académie de musique du Québec

Académie de musique de l'Université du Québec à Trois-Rivières

Société de guitare Claude McKinnon inc.

Conservatoire de musique McGill ${ }^{7}$

École de musique Vincent-d'Indy

École préparatoire de musique Anna-Marie Globenski de l'Université Laval

École préparatoire de musique de l'Université du Québec à Montréal

École préparatoire de musique de l'Université de Sherbrooke

\section{Méthodes analysées}

Des programmes d'enseignement de la guitare de ces organismes, nous avons exclusivement retenu les méthodes suggérées pour l'enseignement aux débutants de o à 2 ans d'expérience. Ainsi, nous avons répertorié 10 méthodes $^{8}$, publiées aux $\mathrm{XX}^{\mathrm{e}}$ et $\mathrm{XXI}^{\mathrm{e}}$ siècles par des auteurs provenant d'Europe, d'Asie et d'Amérique du Nord, dont nous avons analysé le contenu.

$5 \mathrm{http} / / / \mathrm{www}$.mels.gouv.qc.ca/eleves/arts-et-culture/enseignement-prive-de-la-musique/ liste-des-organismes-dencadrement/

6 Par exemple, le piano, le violon, la flûte, le chant, la guitare. Le nombre de programmes et les instruments visés sont à la discrétion des organismes.

7 Bien qu'il en fasse partie depuis de nombreuses années, au moment de publier cet article le Conservatoire de musique McGill ne fait plus partie des organismes reconnus par le MELS.

8 Depuis la constitution du corpus, certaines méthodes on fait l'objet de réédition. Les dates mentionnées dans le tableau réfèrent aux versions analysées, non aux éditions subséquentes disponibles sur le marché. 
Tableau 1. Méthodes analysées

\begin{tabular}{|c|c|c|c|c|c|}
\hline Titre méthode & Auteur & $\begin{array}{c}\text { Nombre de } \\
\text { pages }\end{array}$ & Publication & Public visé & Langue \\
\hline $\begin{array}{l}\text { J'apprends la } \\
\text { guitare } \\
\text { Vol. } 1\end{array}$ & $\begin{array}{l}\text { Marcel Benoît } \\
\text { (1991) }\end{array}$ & 32 & $\begin{array}{l}\text { Éditions M.D. } \\
\text { Inc, Québec }\end{array}$ & $\begin{array}{l}\text { Non } \\
\text { spécifié }\end{array}$ & Français \\
\hline $\begin{array}{l}\text { Méthode } \\
\text { progressive } \\
\text { Vol.1 }\end{array}$ & $\begin{array}{l}\text { Claudio } \\
\text { Camisassa } \\
(2002)\end{array}$ & 64 & $\begin{array}{l}\text { Productions } \\
\text { d'Oz, } \\
\text { Saint-Romuald, } \\
\text { Québec }\end{array}$ & $\begin{array}{l}\text { Non } \\
\text { spécifié }\end{array}$ & $\begin{array}{l}\text { Français } \\
\text { Anglais } \\
\text { Espagnol }\end{array}$ \\
\hline $\begin{array}{l}\text { Modern } \\
\text { Approach to } \\
\text { Classical Guitar } \\
\text { Book } 1\end{array}$ & $\begin{array}{l}\text { Charles Duncan } \\
(1996)\end{array}$ & 63 & $\begin{array}{l}\text { Hal Leonard, } \\
\text { Milwaukee }\end{array}$ & $\begin{array}{l}\text { Non } \\
\text { spécifié }\end{array}$ & Anglais \\
\hline $\begin{array}{l}\text { Guitar Method } \\
\text { for Children }\end{array}$ & $\begin{array}{l}\text { Masaru Koyama } \\
\text { (1975) }\end{array}$ & 71 & $\begin{array}{l}\text { Zen-On Music, } \\
\text { Tokyo }\end{array}$ & $\begin{array}{l}5-6 \text { ans } \\
\text { (p. 1) }\end{array}$ & $\begin{array}{l}\text { Japonais } \\
\text { Anglais }\end{array}$ \\
\hline $\begin{array}{l}\text { Méthode de } \\
\text { guitare pour } \\
\text { débutants } \\
\text { Vol.1 }\end{array}$ & $\begin{array}{l}\text { Sylvain Lemay } \\
(1990)\end{array}$ & 43 & $\begin{array}{l}\text { Productions } \\
\text { d'Oz, } \\
\text { Saint-Romuald }\end{array}$ & $\begin{array}{l}\text { Non } \\
\text { spécifié }\end{array}$ & Français \\
\hline $\begin{array}{l}\text { J'apprivoise la } \\
\text { guitare }\end{array}$ & $\begin{array}{l}\text { Claude McKin- } \\
\text { non } 1 \\
(1984)\end{array}$ & 45 & $\begin{array}{l}\text { Éditions } \\
\text { Geneviève, } \\
\text { Charlesbourg }\end{array}$ & $\begin{array}{l}3 \text { à } 6 \text { ans } \\
\text { (couverture) }\end{array}$ & Français \\
\hline $\begin{array}{l}\text { Mes premiers } \\
\text { pas à la guitare } \\
\text { vol } 1\end{array}$ & $\begin{array}{l}\text { Claude McKin- } \\
\text { non } 2 \\
(2004)\end{array}$ & 67 & $\begin{array}{l}\text { Éditions } \\
\text { Geneviève, } \\
\text { Charlesbourg, } \\
1984\end{array}$ & $\begin{array}{l}\text { De tous } \\
\text { âges dès } 7 \\
\text { ans } \\
\text { (p. 4) }\end{array}$ & Français \\
\hline $\begin{array}{l}\text { New Dimensions } \\
\text { in Classical: Gui- } \\
\text { tar for Children }\end{array}$ & $\begin{array}{l}\text { Sonia Michelson } \\
\text { (1991) }\end{array}$ & 97 & Mel Bay, Pacific & 4-10 (p. 5) & Anglais \\
\hline $\begin{array}{l}\text { Classic Guitar } \\
\text { Techniques, } \\
\text { vol. } 1\end{array}$ & $\begin{array}{l}\text { Aaron Shearer } \\
(1987)\end{array}$ & 120 & $\begin{array}{l}\text { Alfred Music } \\
\text { Publishing, Van } \\
\text { Nuys, Californie }\end{array}$ & $\begin{array}{l}\text { Non } \\
\text { spécifié }\end{array}$ & Anglais \\
\hline $\begin{array}{l}\text { Je deviens guita- } \\
\text { riste vol. } 1\end{array}$ & $\begin{array}{l}\text { Thierry } \\
\text { Tisserand } \\
(2004)\end{array}$ & 32 & $\begin{array}{l}\text { Éditions Henry } \\
\text { Lemoine, Paris }\end{array}$ & $\begin{array}{l}\text { Débutant } \\
\text { en première } \\
\text { année (p. 1) }\end{array}$ & Français \\
\hline
\end{tabular}

Pour analyser le contenu du corpus de méthodes, nous avons utilisé les six étapes proposées par L'Écuyer (1990) concernant l'analyse de contenu.

\section{Thèmes et catégories retenus}

La première étape, lecture préliminaire, (L'Écuyer 1990, p. 57), nous a permis d'obtenir une vue d'ensemble du corpus et nous a servi à "appréhender certaines grandes particularités» (Ibid., p. 58) pouvant constituer des thèmes ou des catégories. Par conséquent, pour répondre à notre question de recherche, nous avons voulu déterminer ce qui est enseigné. Nous avons constaté que les apprentissages proposés aux élèves débutants concernent différents aspects du jeu de la guitare que nous avons regroupés ainsi: 


\section{Posture et tenue de l'instrument}

Ce thème concerne la manière de s'assoir pour bien tenir l'instrument, les accessoires utilisés pour le jeu (pose-pied, lutrin), la bonne tenue générale de la guitare, le positionnement des mains et la détente. Nous avons constaté rapidement que ces aspects étaient présentés dans des proportions très différentes d'une méthode à l'autre.

\section{Techniques de jeu}

Les techniques de jeu concernent isolément soit la main qui pince les cordes pour produire le son (la main droite), soit la main gauche, qui change la hauteur des sons, ou encore les mouvements simultanés des deux mains, essentiels pour jouer une gamme ${ }^{9}$ par exemple. Notons que pour la production du son à la guitare, les deux mains ont des fonctions distinctes. Alors qu'une main permet l'émission du son en attaquant la corde, ce qui représente un type de mouvement, l'autre main change la hauteur des sons sur le manche, avec des mouvements très différents de l'autre main. La synchronisation des mouvements des deux mains permet toute la variété d'attaques et de hauteurs de son de l'instrument. Pour plus de commodité, nous utiliserons la main droite pour désigner la main qui attaque les cordes, bien que certains guitaristes gauchers tiennent l'instrument de manière inversée.

\section{Notes et rythmes utilisés}

Dès la première lecture du corpus, nous avons constaté que bien que l'ordre d'apprentissage des notes soit présenté de manière différente d'une méthode à l'autre, quelques ressemblances s'observent. Ainsi, nous avons cherché à relever les patrons d'apprentissage des premières notes et les rythmes utilisés.

\section{Types d'activités proposées}

Ce thème nous permet d'identifier de quelle manière sont présentés les apprentissages proposés: sont-ils présentés à l'aide d'exercices, de mélodies, de duos, de pièces polyphoniques ou d'exercices de création, avec ou sans lecture de notes? Nous avons relevé la proportion respective de leur présence, le type de répertoire proposé et les tonalités utilisées.

\section{Aspects qualitatifs du jeu}

Nous avons regroupé sous ce grand thème les catégories suivantes: la qualité du son, les nuances, le phrasé, le legato, le senti rythmique, bref ce qui touche à l'expressivité. Ces éléments, qui vont de soi pour le musicien professionnel, ne sont pas toujours présents dans les méthodes. Ainsi, il nous apparaissait important de pouvoir les identifier. 


\section{Notions théoriques}

Différents apprentissages ne concernent pas au premier chef des apprentissages psychomoteurs, mais bien des connaissances plus théoriques, telles que le nom des notes sur la portée et la théorie musicale, des explications sur les parties de la guitare, la manière de bien travailler ou de mémoriser efficacement.

\section{Unités de classification}

La deuxième étape d'analyse de contenu selon L'Écuyer (1990, p. 59) consiste à déterminer le choix et la définition des unités de classification. Ainsi, nous voulions noter la présence ou l'absence des différentes unités de classification sous forme d'unités d'apprentissage "qui vise le développement d'habiletés chez le sujet» (Legendre 2005 p. 1423). Par exemple, un type d'attaque à la main droite, une nouvelle note ou une nuance présentée pour la première fois constituent des unités d'apprentissage. Les unités d'apprentissage font l'objet de «séquences d'activité d'apprentissage» (Ibid., p. 1424) comme des exercices ou des œuvres musicales. En général, les unités d'apprentissage sont bien identifiées dans les méthodes par des titres ou des phrases d'introduction. Il faut cependant noter que ce que les auteurs présentent comme une unité d'apprentissage comporte souvent plusieurs éléments. Prenons l'exemple de l'introduction des trois cordes ouvertes aiguës, $m i$, si et sol. Certains auteurs introduisent le jeu des trois notes dans un seul exercice qui exige la lecture des notes, alors que d'autres pédagogues les présentent individuellement avec différents exercices. Dans le premier cas, l'unité d'apprentissage comprendra les trois notes, et dans le deuxième cas, chaque note fera l'objet d'une unité d'apprentissage qui sera accompagnée de plusieurs activités d'apprentissage.

Ainsi, nous avons déterminé qu'il fallait noter la présence ou l'absence d'éléments précis qui représentent des unités d'apprentissage, mais aussi leur ordre d'arrivée dans la séquence pédagogique proposée pour comprendre la logique didactique qui explique ces choix. Nous avons décidé de répertorier l'apparition des nouvelles unités d'apprentissage avec un codage ordinal (unité de numération de Bardin 2003, p. 60) directement dans les méthodes à l'aide d'un onglet collé sur la page d'apparition afin de pouvoir y accéder aisément.

Nous avons décidé que toutes les unités d’apprentissage proposées dans une méthode, qu'elles soient relatives aux techniques de jeu (une nouvelle attaque), aux aspects qualitatifs (une nuance), aux activités proposées, aux notions théoriques, aux notes et rythmes utilisés, seraient identifiées selon leur ordre d'apparition directement dans les méthodes, puis ensuite reportées sur une grille d'analyse papier propre à chaque méthode analysée. Nous avons décidé de noter les éléments faisant partie de l'introduction des méthodes, souvent en lien avec les notions théoriques, de 0,01 à 0,9 , et les éléments présents dans le corps des ouvrages, à partir de 1 . Ceci nous permet de répondre à la question suivante: qu'est-ce qui est enseigné et dans quel ordre?

Outre l'intérêt de noter l'ordre d'apparition des unités d'apprentissage, il nous apparaissait important d'identifier la manière dont elles sont présentées en détaillant les moyens utilisés. Par exemple, si le premier apprentissage 
proposé dans une méthode est de pincer une corde avec l'index et le majeur en alternance (technique de main droite), il est important de savoir si ces attaques doivent être effectuées sur une corde à vide (seulement avec la main droite) ou fermée (avec la main gauche qui produit la hauteur de son désirée) et si cet apprentissage doit s'effectuer en lisant les notes sur la partition. De plus, les auteurs des méthodes peuvent mettre en place différentes activités autour de ces unités, allant de simples exercices sans lecture à vue à l'exécution d'une œuvre musicale. En effet, une méthode qui propose un apprentissage moteur de l'alternance des doigts sans avoir recours à la lecture de notes et sans y ajouter la synchronisation des deux mains sur le manche pour la production de notes repose sur un paradigme pédagogique différent d'une méthode qui combine tous ces éléments dans une seule activité d'apprentissage plus complexe, prenant plutôt forme dans une nouvelle œuvre à jouer. Ceci nous a permis de répondre à la question: de quelle manière et avec quels types d'exercices ou de répertoire sont présentés les éléments à faire apprendre dans le matériel éducatif utilisé par les enseignants?

Nous avons décidé de confectionner la grille d'analyse de manière à pouvoir combiner la présence ou l'absence d'unité d'apprentissage en les identifiant par un chiffre qui représente leur ordre d’arrivée des séquences d’apprentissage dans cette grille. Finalement, nous avons précisé avec quel type d'activités (exercices, pièces) les unités d'apprentissage sont présentées.

\section{Processus de catégorisation}

La troisième étape proposée par L'Écuyer (1990, p. 63) pour l'analyse de conte$\mathrm{nu}$, c'est-à-dire le processus de catégorisation, doit permettre une réorganisation des informations en catégories pour en effectuer une analyse. Comme la qualité de l'analyse repose en grande partie sur les catégories utilisées, nous avons porté une attention particulière à cette étape en procédant à une validation auprès d'experts.

Nous avons développé une première grille mixte, avec des catégories préexistantes et la possibilité d'ajouter d'autres catégories grâce à l'analyse de cinq ouvrages par la chercheuse. Par la suite, l'analyse croisée des dix ouvrages par la chercheuse et une assistante de recherche ${ }^{10}$ a permis d'augmenter le nombre de catégories pour finalement constituer une grille fermée avec une légende qui définit les items présents. Pour terminer l'élaboration de cette grille d'analyse, pallier les biais idéologiques ou affectifs (Van der Maren 1996, p. 119), nous assurer de l'objectivité, de la clarté, de l'exhaustivité, de la pertinence des catégories (L'Écuyer 1990, p. 83) et des éléments qui y seront recueillis, nous avons procédé à une validation auprès de deux experts. Les commentaires et recommandations de Peter McCutcheon et de Jean Vallières, respectivement professeurs à l'Université de Montréal et au Conservatoire de Musique de Montréal, sont incorporés à la version 11 de la grille de modèle fermé, dont toutes les catégories étaient prédéterminées. Cette grille a servi à l'analyse de contenu finale.

10 Le but visé était d'obtenir un nombre d'entrées et un ordre similaire à + ou -1 ou 2 erreurs pour un accord inter-juges acceptable. 
Ainsi, nous nous sommes assurée que les catégories soient pertinentes au jeu de la guitare, qu'elles tiennent compte des spécificités de l'instrument (mouvements différents de chacune des mains et mouvements simultanés) et qu'elles rendent compte des étapes d'apprentissage qui sont généralement identifiées clairement dans les programmes d'enseignement et dans les méthodes ellesmêmes (par exemple, le début du jeu polyphonique, les premiers changements de position). De plus, ces catégories nous informent sur les types d'activités proposées pour les apprentissages (pièces, exercices et tonalités). Nous avons donc analysé les dix méthodes avec une version imprimée de cette grille finale (vous en trouverez un extrait à l'Annexe 1).

\section{Quantification et traitement statistique}

En accord avec la quatrième étape proposée par L'Écuyer (1990, p. 101), nous avons procédé à des analyses quantitatives descriptives des données recueillies sur les grilles papier développées à la troisième étape et qui ont servi à analyser les méthodes de notre corpus. Par conséquent, nous avons constitué une base de données avec le logiciel Excel 2003 dans laquelle la méthode a fait l'objet d'une feuille de classeur. L'axe horizontal de la feuille de classeur Excel permet de noter l'ordre d'apparition des unités d'apprentissage, qu'elles soient isolées ou combinées à d'autres, dans la colonne correspondant à leur ordre d'arrivée dans le déroulement de la méthode. Ainsi, chaque nouvelle unité d'apprentissage introduite dans une méthode qui figure sur l'axe vertical (par exemple, une nouvelle note ou une nouvelle technique) a été notée par le chiffre de son ordre d'arrivée dans la colonne correspondante de la feuille Excel. Elle peut ainsi être combinée avec d'autres informations figurant aussi sur l'axe vertical par simple identification du chiffre correspondant à son ordre d'arrivée au bon endroit. L'axe vertical proposait 181 possibilités d'entrées (notes, précisions sur les techniques, quantité d'exercices, etc.) qui peuvent être combinées entre elles et ainsi nous informer sur le contenu de chacun des éléments d'apprentissage identifié. Ainsi, au-delà de la simple identification de la présence d'un apprentissage particulier dans une méthode (ou de plusieurs apprentissages simultanés), nous pouvons déterminer le moment de son introduction dans la séquence d'apprentissage, la manière dont il a été introduit, de même que le nombre et le type d'activités proposées pour ce nouvel apprentissage. Vous pouvez consulter un extrait de la feuille de classeur pour la méthode J'apprivoise la guitare, de McKinnon (1984) à l'Annexe 1.

\section{Description scientifique et interprétation des résultats}

Pour la cinquième étape, soit la description scientifique (L'Écuyer 1990), nous avons procédé à une analyse qualitative des données grâce aux statistiques descriptives obtenues à l'étape précédente. Parmi l'ensemble des résultats, nous avons mis en exergue ceux pouvant répondre à notre question générale de recherche: quels sont les paradigmes didactiques qui guident l'enseignement de la guitare classique aux débutants de la province de Québec? Les résultats 
de l'analyse de contenu des méthodes analysées sont présentés dans la section Résultats.

Pour la sixième étape de l'analyse de contenu, l'interprétation des résultats, L'Écuyer note que «Selon les d'objectifs visés, l'étape de la description scientifique (quantitative et qualitative) peut être suivie d'une dernière étape tirée soit directement des analyses quantitatives ou qualitatives, soit l'analyse symbolique, soit en références à des construits ou modèles théoriques » (p. 301). Dans le cas de cet article, l'interprétation des résultats sera présentée dans la section Discussion qui a pour but d'identifier les paradigmes présents dans notre corpus. Il nous a fallu garder en tête les limites de cette étude: le nombre restreint de méthodes analysées et les limites des données que nous avons recueillies dans cette étude exploratoire.

\section{RÉSULTATS}

Nous présentons ici les résultats de l'analyse des dix méthodes de notre corpus. Rappelons que ces méthodes sont proposées par les organismes d'encadrement de la province de Québec pour l'enseignement de la guitare aux débutants.

\section{Âge visé par les méthodes}

Dans un premier temps, nous avons voulu connaître l'âge des apprenants visés. Comme certains auteurs ne précisent pas l'âge du public cible (voir Tableau 1), nous devons le déduire grâce à différents indices tels que les éléments visuels présents et la complexité du langage utilisé pour les consignes. McKinnon 1 (1984) est l'auteur qui veut favoriser l'apprentissage de la guitare chez les enfants les plus jeunes, soit de trois à six ans. Sa méthode est illustrée de dessins rudimentaires réalisés à la main, représentant des animaux ou des formes comme un soleil, avec de courtes explications écrites dans un langage simple et accessible qui s'adresse directement aux petits. Des photos en noir et blanc d'une enfant d'environ cinq ans illustrent la position à adopter à la guitare. Michelson (1991) vise l'enseignement de la guitare aux enfants de quatre à dix ans, mais sa méthode s'adresse spécifiquement aux enseignants et aux parents avec des explications sur les apprentissages proposés, des consignes d'enseignement et des exercices complémentaires d'éveil musical. Elle propose quelques photos, tout comme celle de Koyama (1975) qui vise les enfants âgés de cinq ans et plus, et s'adresse aussi aux parents et aux enseignants. Tisserand (2004) indique que sa méthode s'adresse aux «débutants en première année», ce qui peut être ambigu. Cependant, les dessins colorés qui illustrent la méthode tout au long, dont ceux d'un garçon représenté à maintes reprises, nous indiquent bien qu'il est question d'enfants de première année du primaire. Cette méthode est la seule qui soit abondamment illustrée et imprimée en couleurs. Les commentaires écrits sont adressés aux enfants dans un langage simple. À l'exception de celle de Koyama, ces méthodes offrent essentiellement du répertoire monodique.

La méthode de McKinnon 2 (2004) s'adresse spécifiquement aux débutants de tous les âges, à partir de sept ans. Sans illustration, sinon la photo d'une jeune femme pour démontrer la posture, elle offre des sections avec des 
exercices techniques et des explications simples, mais non infantilisantes. Bien quaucune indication ne soit donnée par les auteurs au sujet du public cible, les méthodes de Duncan (1996), Benoît (1991) et Lemay (1990) semblent s'adresser à la même tranche d'âge. Si les explications sont plus nombreuses chez Duncan (1996) et Benoît (1991) et laissent présager du fait que ces méthodes s'adressent autant à des adultes qu'à des enfants, elles sont pratiquement inexistantes chez Lemay (1990).

Camisassa (2002) ne spécifie pas l'âge des apprenants visés. Par contre, la photo d'une fillette d'environ dix ans laisse deviner qu'il s'agit d'une méthode pour enfants. Les explications données en introduction et celles qui ponctuent la méthode confirment que l'auteur s'adresse à des enfants de la fin du primaire et du début de l'école secondaire. Il s'agit du seul ouvrage présenté en trois langues. Les photos qui illustrent la méthode de Shearer (1987) mettent en scène l'auteur et un adulte d'une trentaine d'années et les nombreuses explications fournies en début et en fin des méthodes ne laissent planer aucun doute sur la clientèle visée.

\section{Le corps dans le jeu}

Le tableau 2 détaille les résultats obtenus pour tout ce qui touche à la posture et à la tenue de l'instrument. Les chiffres désignent à quel moment les informations apparaissent dans les méthodes. Ainsi, un élément noté 5 indique qu'il est présenté avant l'élément 6 dans la méthode. Nous avons divisé ce qui figure dans l'introduction des méthodes $(0,01)$, de ce qui est placé dans le cour des méthodes $(1,2,3)$ pour refléter la structure donnée par les auteurs à leur ouvrage

Deux méthodes, soit celle de Benoît (1991) et de Lemay (1990) n'abordent ni la posture ni la tenue de l'instrument. Michelson (1991) mentionne l'importance d'une bonne posture assise, mais sans plus. La plupart des auteurs présentent la posture comme une position statique, soit à l'aide d'illustrations fantaisistes pour Tisserand (2004) ou de photos prises selon différents angles pour Shearer (1987), voire carrément sur un seul plan pour les autres ouvrages.

En ce qui concerne le bras, la main et les doigts du côté droit, celui qui pince les cordes, nous remarquons que Camisassa (2002), Koyama (1975), McKinnon 1 (1984) et McKinnon 2 (2004) proposent leurs consignes dans l'introduction ou au début de leur méthode. Duncan (1996), Benoît (1991), Michelson (1991), Shearer (1987), et dans une moindre mesure Tisserand (2004) et Michelson (1991), sont préoccupés par la stabilité et la détente des membres avec des consignes qui apparaissent régulièrement tout au long de la méthode. Duncan (1996) et surtout Shearer (1987) sont les deux auteurs qui présentent le plus de consignes relatives à la position des mains et des doigts. La position du bras, des mains, des doigts et plus particulièrement du pouce derrière le manche n'est pas abordée par Benoît (1991), Lemay (1990), McKinon (1984) 1 et McKinnon 2 (2004), alors que Camisassa (2002) et Koyama (1975) en traitent uniquement dans l'introduction. Michelson (1991) aborde plus souvent les éléments de la main qui touchent le côté gauche du corps, contrairement à Shearer (1987) qui semble accorder moins d'attention au côté gauche quau droit. La méthode 


\begin{tabular}{|c|c|c|c|c|c|c|c|c|c|c|c|c|c|c|c|c|c|c|c|}
\hline & \multicolumn{2}{|c|}{ Posture } & \multicolumn{6}{|c|}{ Main droite } & \multicolumn{11}{|c|}{ Main gauche } \\
\hline & 음 & 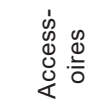 & 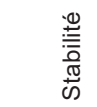 & $\begin{array}{l}0 \\
\stackrel{0}{0} \\
\stackrel{0}{0} \\
0 \\
0\end{array}$ & 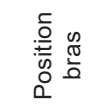 & 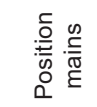 & $\begin{array}{l}\frac{}{0} \\
: \frac{0}{5} \\
\frac{+}{0} \\
0 \\
0\end{array}$ & $\begin{array}{l}\text { D } \\
\frac{0}{0} \\
0\end{array}$ & 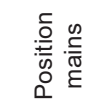 & $\begin{array}{l}\frac{5}{0} \\
\frac{0}{ \pm} \\
\frac{5}{0} \\
0 \\
0 \\
0\end{array}$ & 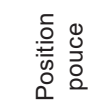 & $\begin{array}{l}\frac{5}{0} \\
: \frac{0}{ \pm} \\
\frac{\pi}{0} \\
0 \\
0\end{array}$ & 흠 Ð & 흥. & 흥 음 & & & & 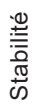 \\
\hline $\begin{array}{l}\text { Marcel Benoît } \\
\text { (1991) }\end{array}$ & & & $\begin{array}{l}1-10- \\
12-16- \\
17\end{array}$ & $1-16-17$ & & & & 3 & & & & & $5-8$ & & & & & & \\
\hline $\begin{array}{l}\text { Claudio } \\
\text { Camisassa } \\
(2002)\end{array}$ & 0,01 & 0,01 & 1 & & 0,03 & 0,03 & 0,03 & & $0,03-2$ & $0,03-2$ & 0,03 & 0,01 & & 2 & & 0,01 & & 2 & \\
\hline $\begin{array}{l}\text { Charles Duncan } \\
\text { (1996) }\end{array}$ & 0,03 & 0,03 & $\begin{array}{l}2-9- \\
10-11- \\
25\end{array}$ & 1 & 0,05 & $\begin{array}{l}0,05-1- \\
9-10-11\end{array}$ & $\begin{array}{l}0,05-1- \\
2-9-10- \\
11-12\end{array}$ & 3 & $14-15$ & $14-15$ & $14-15$ & 14 & $\begin{array}{l}16-22- \\
26\end{array}$ & $22-28$ & & 14 & & & \\
\hline $\begin{array}{l}\text { Masaru Koyama } \\
\text { (1975) }\end{array}$ & 0,02 & 0,02 & & 0,03 & 0,02 & 0,03 & 0,03 & 0,09 & 0,03 & 0,03 & 0,03 & & 2 & & & & & & \\
\hline $\begin{array}{l}\text { Sylvain Lemay } \\
(1990)\end{array}$ & & & & & & & & & & & & & $5-8-10$ & & & & & & \\
\hline $\begin{array}{l}\text { Claude McKinnon } \\
1(1984)\end{array}$ & 0,02 & 0,02 & & & 0,03 & 0,03 & 1 & & & & & & & $6-7$ & $\begin{array}{l}5-6- \\
7-8\end{array}$ & & & & \\
\hline $\begin{array}{l}\text { Claude McKinnon } \\
2(2004)\end{array}$ & $0,03-1$ & $0,03-1$ & & & $0,04-1$ & $0,04-1$ & $0,04-1$ & & & & & & & 14 & $\begin{array}{l}8-9- \\
10-14\end{array}$ & & & $\begin{array}{l}8- \\
16\end{array}$ & \\
\hline $\begin{array}{l}\text { Sonia Michelson } \\
\text { (1991) }\end{array}$ & 0,01 & & $4-8$ & & & $0,01-8$ & & & $0,01-8$ & $\begin{array}{l}6-8-9- \\
14-16\end{array}$ & & & $\begin{array}{l}7-8- \\
9-11- \\
13-14\end{array}$ & 9 & & & & & \\
\hline $\begin{array}{l}\text { Aaron Shearer } \\
\text { (1987) }\end{array}$ & 0,07 & 0,07 & $\begin{array}{l}0,08- \\
2-8-9- \\
11-15- \\
16-17- \\
22-27- \\
29-31\end{array}$ & $\begin{array}{l}0,08- \\
2-15- \\
16-20- \\
21-22- \\
27-31\end{array}$ & $\begin{array}{l}0,07- \\
9-12\end{array}$ & $\begin{array}{l}0,08- \\
2-6-8- \\
9-15- \\
16-20- \\
21-22\end{array}$ & $\begin{array}{l}0,08- \\
2-6-8- \\
9-12- \\
15-16- \\
20-21- \\
22\end{array}$ & $0,08-12$ & 7 & $7-8-28$ & 7 & 7 & $\begin{array}{l}7-8- \\
13-24- \\
29-31- \\
32\end{array}$ & & $29-32$ & $7-8$ & & 7 & \\
\hline $\begin{array}{l}\text { Thierry Tisserand } \\
(2004)\end{array}$ & 0,03 & 0,03 & $15-16$ & 9 & $0,03-1$ & $0,03-1$ & $\begin{array}{l}0,03-1- \\
15-16\end{array}$ & & 7 & 7 & $7-9-14$ & 0,03 & $\begin{array}{l}10-13- \\
18\end{array}$ & $9-12$ & 14 & & & 14 & \\
\hline
\end{tabular}


de Lemay (1990) traite peu des différents aspects relatifs au corps et présente seulement à trois reprises la consigne de maintenir les doigts en place lors du jeu pour favoriser le legato.

De plus, seules quatre méthodes, celles de Benoît (1991), de Duncan (1996), de Koyama (1975) et de Shearer (1987), abordent le jeu avec les ongles, pourtant essentiel pour l'attaque de la corde à la guitare classique. Comme les méthodes analysées s'adressent majoritairement aux débutants plutôt jeunes, nous pouvons penser que les auteurs considèrent que des explications écrites détaillées seraient inappropriées. Par contre, Michelson (1991) et Koyama (1975), même s'ils s'adressent aux parents et aux enseignants, offrent peu d'informations à ce sujet. Shearer (1987), et dans une moindre mesure Camisassa (2002), proposent cependant des photos pour illustrer les consignes relatives au corps, ce qui permet à l'apprenant de s'y référer facilement. Shearer (1987) est celui qui offre les explications les plus complètes et le plus de photos et d'illustrations, entre autres pour limer les ongles, mais il s'adresse aussi aux débutants plus âgés.

Tous les auteurs formulent des consignes quant au bon placement des doigts dans les cases et à la nécessité de l'alternance des doigts de la main droite. Par exemple, si Tisserand (2004) et Shearer (1987) ne présentent pas d'exercices techniques dans une section spécifique, ils offrent à plusieurs reprises des consignes claires et de courts exercices sur la manière de placer les doigts lorsqu'un nouveau geste est présenté dans leur ouvrage. Par exemple, pour anticiper le jeu d'une nouvelle note, Tisserand (2004, p. 15) donne la consigne suivante: «Je dirige [le doigt] 2 vers [la note] la, pendant [que je joue] le si». Quant à Shearer (1987), il propose des consignes pour une bonne position du pouce $(p)$, pour la tenue des notes et pour le maintien des doigts en place afin d'assurer le legato du jeu (p. 37).

\section{Techniques de jeu}

Cette section présente les premiers apprentissages proposés à la main droite et les différentes techniques de jeu, incluant aussi la main gauche, qui sont abordés dans les ouvrages analysés. L'utilisation de la main gauche pour changer les notes sera abordée dans la section des patrons d'apprentissage des notes. Ici aussi, les chiffrent indiquent l'ordre d'arrivée des éléments dans la méthode.

\section{Jeu monodique et alternance des doigts}

Chez tous les auteurs, le premier moment de jeu proposé à l'instrument est un exercice dans lequel la main droite attaque une corde ouverte (sans le recours à la main gauche). Si cet exercice s'effectue sans lecture de notes dans la plupart des méthodes, Benoît (1991), Koyama (1975), Michelson (1991) et Lemay (1990) introduisent la lecture dès le début du jeu avec des rondes, des blanches ou des noires.

Il existe deux types d'attaques possibles à la main droite, soit butée ou tirée, différenciées par le mouvement qu'effectue le doigt après l'attaque. En effet, après avoir pincé la corde, le doigt peut terminer sa trajectoire en s'appuyant sur la corde adjacente dans le cas de l'attaque butée, ou revenir à sa position 
initiale sans toucher aux autres cordes. McKinnon 1 (1984) et McKinnon 2 (1981), Camisassa (2002) et Michelson (1991), préconisent exclusivement l'usage de l'attaque tirée. Camisassa (2002), Shearer (1987) et Tisserand (2004) proposent de jouer avec les deux attaques dès le départ, alors que Benoît (1977) et Duncan (1996) introduisent l'attaque tirée plus tard dans la séquence d'apprentissage. Lemay (1990) ne spécifie pas le type d'attaque demandé.

Dans le jeu d'une mélodie seule, les doigts qui attaquent les cordes le font en alternance: ainsi, l'index $(i)$ et le majeur $(m)$ pincent la corde l'un après l'autre. Nous pouvons aussi avoir recours à une alternance entre le majeur $(m)$ et l'annulaire $(a)$, entre l'index et l'annulaire ou à des combinaisons à trois doigts telle $i-m-a$. Si l'alternance des doigts $i-m$ dans le jeu monodique est présent chez tous les auteurs, et ce, dès les premières notes jouées et peu importe l'âge visé, on constate la prévalence du jeu monodique avec l'alternance $i-m$ sur le jeu polyphonique pour les jeunes débutants avec des rythmes plus simples. Nous constatons que l'utilisation de l'alternance $m-a$ et $i-a$ est plus rare dans les méthodes analysées et elles le sont à titre d'exercices techniques et non de doigtés à privilégier dans les pièces. Shearer (1987) est le seul qui propose de jouer des notes répétées sur les trois premières cordes ouvertes avec le $a$ pour la première corde, le $m$ pour la deuxième et le $i$ sur la troisième corde, à l'image des doigtés utilisés pour le jeu polyphonique. Shearer (1987) est l'auteur qui introduit le jeu du pouce le plus tôt, suivi de Koyama (1975), de Camisassa (2002), de Michelson (1991) et, plus tardivement dans la séquence pédagogique de leur méthode, de Benoît (1991), de Duncan (1996), de McKinnon 2, de Tisserand (2004) et de Lemay (1990). McKinnon 1 (1984) est le seul qui n’aborde pas le jeu du pouce.

\section{La polyphonie}

Dans le jeu polyphonique, les doigts, disposés sur plusieurs cordes, peuvent jouer simultanément. Le pouce est aussi sollicité. La polyphonie est introduite dans les ouvrages après l'apprentissage du jeu monodique grâce à des techniques de jeu simples, comme l'alternance entre les basses et les cordes aiguës. Shearer (1987), Koyama (1975) et dans une moindre mesure Camisassa (2002) proposent des ouvrages contenant des exercices et du répertoire polyphoniques dès le début des apprentissages et très peu d'exercices au jeu monodique. On remarque aussi que les rythmes utilisés sont simples, particulièrement dans le répertoire polyphonique, sauf chez Koyama (1975) qui utilise des rythmes plus complexes (par exemple la double-croche, p. 35) ainsi que des techniques plus avancées de coulé et de jeu simultané.

Larrivée de la polyphonie est tardive chez Tisserand (2004) et Lemay (1990) qui proposent, en fin de méthode, une section comprenant différentes œuvres à deux voix, où alternent une note basse et une note aiguë pour créer la polyphonie, comme l'illustre l'exemple 1.

Chez Camisassa (2002), si l'utilisation des basses alternées avec les notes de la mélodie est utilisée pour introduire le jeu polyphonique, le jeu des notes simultanées est aussi abordé rapidement. 


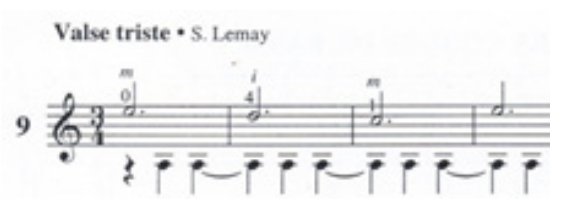

Exemple 1: Lemay (1990, p. 38)

\section{Bourrée}

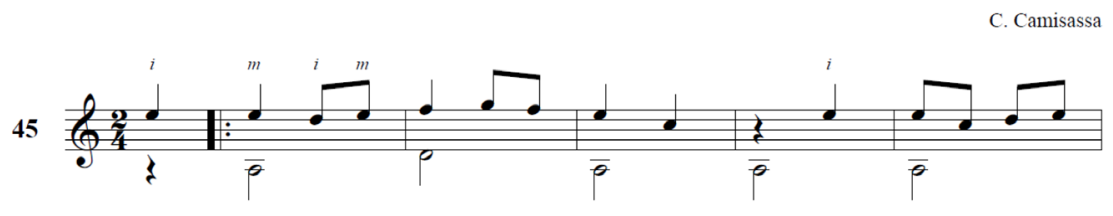

Exemple 2: Camisassa (2002, p. 26)

Chez tous les auteurs de notre corpus qui abordent la polyphonie, ce sont les cordes ouvertes qui sont majoritairement utilisées pour les basses, sauf pour Koyama (1975) qui utilise les cordes fermées à la basse dès le premier morceau.

\section{Rythmes utilisés}

Le tableau 3 nous informe des figures de note et de silence utilisées dans les méthodes. Ainsi, nous voyons dans toutes les méthodes l'utilisation des rondes, des blanches, des blanches pointées et des noires. Seul McKinnon 1 (1984) n'utilise ni les croches ni aucune autre nouvelle figure rythmique. De plus, aucune figure de silence n'est utilisée dans sa méthode. La noire pointée, le soupir, le demi-soupir et la noire liée sont utilisés dans huit méthodes, alors que la demipause est utilisée dans sept méthodes. La double-croche, la croche pointée, la pause, le triolet et la pause pointée sont les figures les moins utilisées. 
Tableau 3. Figures de note et de silence utilisées

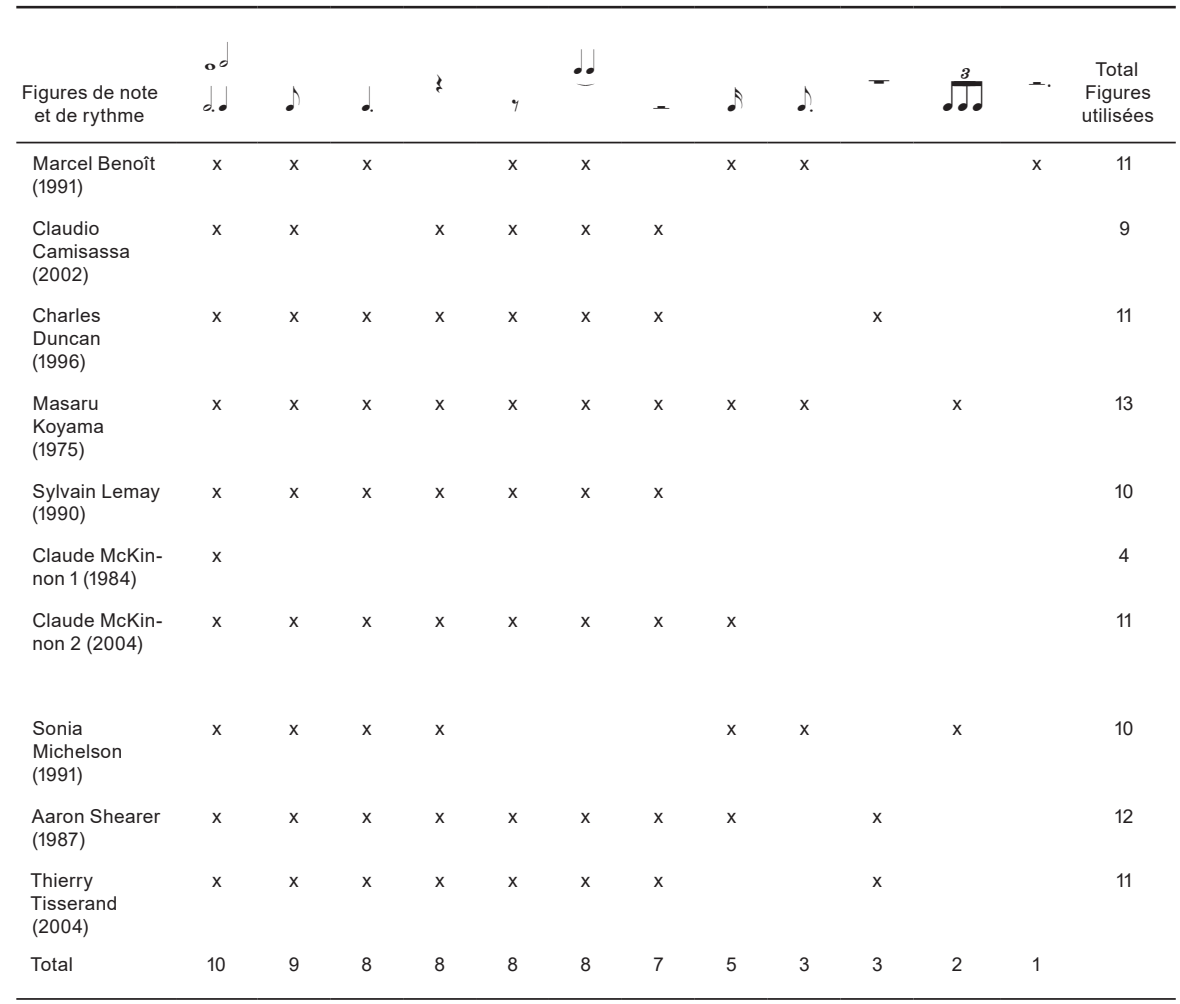

\section{Apprentissage des notes à la guitare: deux approches}

Dans trois méthodes, les deux premières notes apprises sont des cordes ouvertes soit le $m i$ aigu première corde et le si deuxième corde alors que la méthode de Lemay (1990) propose le sol ouvert suivi du la sur la troisième corde avec l'utilisation du deuxième doigt. Lemay (1990) est l'auteur qui introduit la synchronisation entre les deux mains le plus tôt. Michelson (1991) introduit le ré deuxième corde avec l'utilisation du ré troisième doigt après le si corde ouverte.

En examinant les différents patrons d'apprentissage des notes présents dans les méthodes et les activités d'apprentissage proposées (exercices avec ou sans lecture, pièces), nous pouvons identifier deux approches pour l'apprentissage des notes à la guitare. La première approche privilégie le jeu des cordes ouvertes qui implique une seule main dans l'émission du son. Ces méthodes, Duncan (1996), Camisassa (2002) McKinnon 1 (1984), McKinnon 2 (2004), Shearer (1987), Tisserand (2004), proposent plusieurs exercices et petites pièces sur les trois premières cordes ouvertes avant d'introduire le jeu des notes à cordes fermées, qui requiert la synchronisation entre la main gauche qui appuie sur le manche et la main droite qui attaque la corde. 


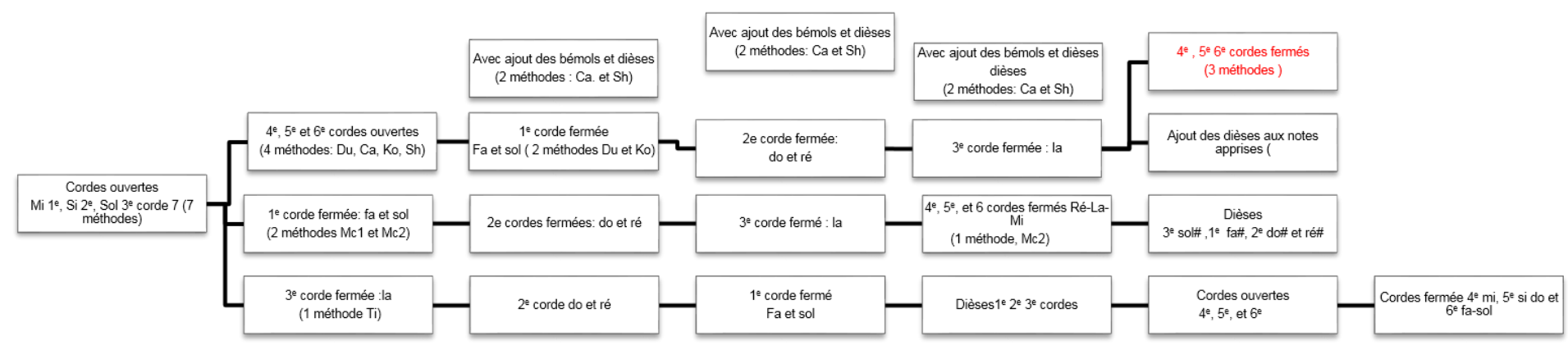


Figure 2. Patrons d'apprentissage avec cordes fermées
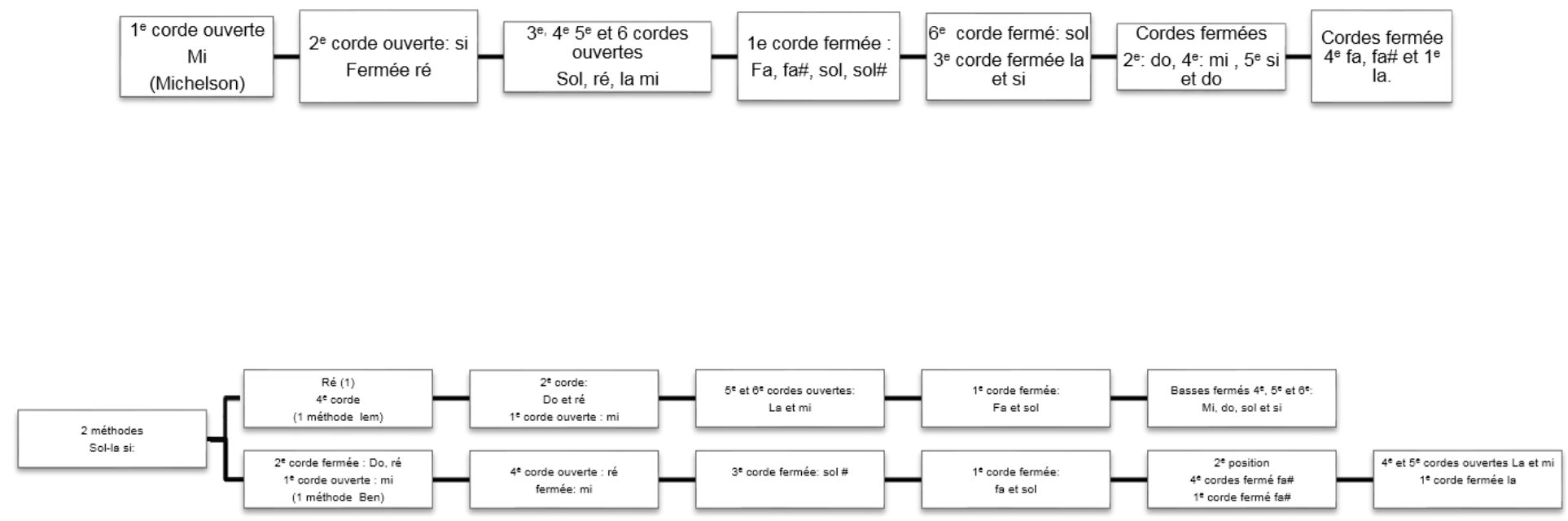
La deuxième approche, celle préconisée par Benoît (1991), Koyama (1975), Lemay (1990) et Michelson (1991), introduit rapidement l'action de la main gauche pour changer la hauteur des sons. Cette approche demande une synchronisation entre les deux mains dès le début du jeu.

\section{SECTIONS DÉDIÉES AUX EXERCICES TECHNIQUES}

\section{Exercices techniques}

Lemay (1990) est le seul à proposer huit gammes à la fin de son volume (p. 43), mais il n'indique pas de consignes de travail. Il faut cependant noter que les organismes d'encadrement, qui proposent les méthodes à utiliser, exigent toujours le jeu de quelques gammes et d'exercices techniques lors des examens de fin d'année. Ainsi, nous pourrions être surpris de constater que seuls Camisassa (2002), Lemay (1990) et McKinnon 2 (2004) proposent des sections contenant des exercices techniques visant le développement de la main gauche et la synchronisation des deux mains. En fait, les programmes d'études des organismes d'encadrement proposent des cahiers de gammes ou d'exercices techniques (Université Laval), ou indiquent clairement les exercices techniques à présenter aux examens à même leur programme (Université de Sherbrooke, UQAM).

Camisassa (2002), Lemay (1990) et McKinnon 2 (2004) proposent des sections contenant des exercices techniques visant le développement de la main gauche et la synchronisation des deux mains. Camisassa (2002) y consacre trois pages (p. 4, 5 et 7), tout comme McKinnon 2 (p. 17, 34 et 56), tandis que Lemay (1990) n'y consacre qu'une page (p. 42). De plus, Camisassa (2002) introduit un exercice de mouvement contraire en cours de méthode (p. 44). Lemay (1990) est le seul auteur qui ne donne pas de consignes sur la manière d'effectuer les exercices. Koyama (1975) présente des exercices techniques selon les besoins du répertoire, soit des demi-barrés ${ }^{11}$, des barrés complets (p. 49) ainsi que des coulés ${ }^{12}$ (p. 54).

Benoît (1991), Camisassa (2002), McKinnon 1 (1984) et 2 (2004) et Michelson (1991) ne proposent aucun exercice de gammes, alors que les autres auteurs le font dans des proportions différentes et avec des objectifs divers. En effet, pour certains auteurs les gammes proposées ont pour objectif de faire apprendre le nom des notes. Pour d'autres, elles servent d'exercices favorisant le développement de la technique instrumentale, mais elles peuvent aussi servir les deux objectifs. Shearer (1987) propose deux exercices avec la gamme chromatique sur les trois premières cordes en première position pour travailler la synchronisation (p. 52, 61 et 75). Tisserand (2004) propose deux gammes à une octave

11 La technique du barré consiste à utiliser l'index de la main gauche pour appuyer simultanément sur plusieurs cordes dans une même case afin d'en changer la hauteur. Lorsque l'index appuie sur les six cordes, il s'agit d'un barré complet. Si le doigt appuie sur plus de deux cordes, mais moins que six, il s'agit d'un demi-barré ou barré partiel. Cette technique exige de la force et un bon contrôle de la pression exercée sur le manche.

12 Le coulé est une technique permettant d'attaquer une note avec la main gauche seule dans un effet percussif, sans le recours à la main droite pour actionner la corde. Cette technique exige de la force et beaucoup de précision. 
en première position et sur les trois premières cordes, dont une dans le mode de sol pour travailler l'alternance (p. 16), et une chromatique à titre de récapitulatif des notes apprises (p. 32). Duncan (1996) propose trois gammes à une octave en première position: une gamme de do majeur sans consigne particulière sinon le respect des doigtés, une première gamme chromatique sur les trois cordes aigües et une seconde débutant sur le la cinquième corde avec le but avoué de développer le contrôle et la force des doigts. La dernière gamme est aussi présentée comme un échauffement "for practice warm-up» (p. 59). Koyama (1975) propose des exercices de gammes sur une octave en six tonalités (p. 61). Si les gammes de mi majeur et de mi mineur mélodique sont en première position, les gammes de la majeur et de la mineur mélodique sont en deuxième position et les gammes de ré majeur et de ré mineur mélodique débutent en deuxième position et impliquent un déplacement en septième position pour atteindre le ré de la première corde. Elles visent la connaissance des notes et une certaine aisance dans le jeu que l'on retrouve dans les tonalités souvent utilisées à la guitare et doivent être travaillées avec attention.

Lemay (1990) consacre une page complète (p. 43) aux gammes en première position et en deuxième position pour les gammes de la majeur et de la mineur mélodique. Les gammes de do majeur, de ré majeur et de ré mineur mélodique sont sur une octave, et les gammes de la majeur et de la mineur mélodique, de sol majeur, de $m i$ mineur mélodique et de $f a$ majeur sont sur deux octaves. Les doigtés de la main gauche sont proposés, mais aucune consigne n’est donnée.

\section{Accords}

Le jeu des accords, permettant l'accompagnement d'une mélodie, n'est pas abordé dans la majorité des ouvrages. Shearer (1987) utilise l'accord de mi majeur comme moyen de vérifier l'accordage de la guitare (p. 13). Michelson (1991), dont l'ouvrage s'adresse aux enfants de trois ans et plus, présente des accords simples à cordes ouvertes, mi mineur, la mineur et ré en accord de septième, ainsi que les accords de do majeur et de sol majeur simplifiés. Il préconise l'utilisation des trois premières cordes, dans une section dédiée à l'accompagnement de chansons simples (p. 67), avec le pouce qui brosse les trois premières cordes. Duncan (1996) présente les accords de la mineur et de mi et ré majeurs avec trois pièces à accompagner. Il propose de brosser les cordes avec le pouce, mais aussi d'effectuer un accompagnement avec une alternance entre la basse, jouée avec le pouce, et l'aigu $i, m, a$, en noires. Camisassa (2002) est l'auteur qui accorde le plus d'importance aux accords. En effet, il les introduit dès le début du jeu, au cinquième élément d'apprentissage, pour accompagner les premiers exercices sur des cordes ouvertes. Ainsi, mi mineur et la majeur sont les premiers accords introduits (p. 8), suivis de do majeur et sol majeur (p. 10), ré en accord de septième, ré majeur, la majeur (pp. 10 et 11), ré mineur et mi majeur (p. 17), mi en accord de septième (p. 20), fa en accord de septième avec un grand 
barré13 ré mineur 4 (p.24). Finalement, les accords de mi mineur diminué, sol mineur/si bémol et le la en accord de septième sont introduits à la page 41.

\section{Types d'activités proposées}

Le tableau suivant nous présente le nombre d'exercices, de mélodies (monodies pouvant être accompagnées par l'enseignant ou par un disque compact) et de pièces (polyphonies, c'est-à-dire au moins deux voix, une mélodie accompagnée par une basse jouée à une seule guitare) et le pourcentage que cela constitue dans chacune des méthodes. Nous avons additionné le nombre d'exercices, de mélodies et de pièces afin d'obtenir le nombre d'items à jouer qui sont proposés aux élèves dans chacune des méthodes. Nous avons aussi indiqué le nombre d'éléments d'apprentissage et le nombre de pages que comporte chaque méthode. Nous désirions savoir si certaines méthodes présentent plus ou moins d'items à jouer par éléments d'apprentissage. Pour ce faire, nous avons déterminé un indice de densité (Tableau 4) en divisant simplement le nombre d'items par le nombre d'éléments d'apprentissage présentés

Tableau 4. Types d'activité proposée

\begin{tabular}{|c|c|c|c|c|c|c|c|c|c|c|}
\hline & \multicolumn{2}{|c|}{ 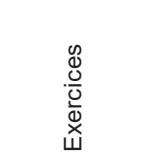 } & \multicolumn{2}{|c|}{$\begin{array}{l}\frac{0}{\partial} \\
\frac{0}{0} \\
\sum\end{array}$} & \multicolumn{2}{|c|}{ 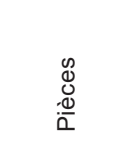 } & \multirow{2}{*}{ 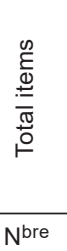 } & \multirow{2}{*}{ 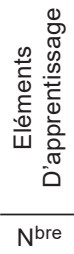 } & \multirow[t]{2}{*}{ 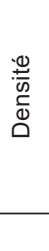 } & \multirow{2}{*}{ 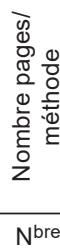 } \\
\hline & $\mathrm{N}^{\text {bre }}$ & $\%$ & $N^{\text {bre }}$ & $\%$ & $\mathrm{~N}^{\text {bre }}$ & $\%$ & & & & \\
\hline $\begin{array}{l}\text { Benoît } \\
\text { (1991) }\end{array}$ & 46 & 78 & 16 & 27 & 13 & 22 & $59^{a}$ & 21 & 3,6 & 32 \\
\hline Camisassa (2002) & 72 & 55 & 13 & 10 & 45 & 35 & $130^{a}$ & 16 & 8,1 & 64 \\
\hline Duncan (1996) & 80 & 63 & 40 & 31 & 8 & 6 & $128 * a$ & 29 & 4,8 & 63 \\
\hline Koyama (1975) & 53 & 51 & 14 & 14 & 36 & 35 & $103^{a}$ & 16 & 6,4 & 71 \\
\hline Lemay (1990) & 87 & 59 & 52 & 35 & 9 & 6 & $148^{*}$ & 18 & 8,2 & 43 \\
\hline McKinnon 1 (1984) & 33 & 69 & 15 & 31 & 0 & 0 & $48^{a}$ & 8 & 6,0 & 45 \\
\hline McKinnon 2 (2004) & 53 & 55 & 24 & 25 & 19 & 20 & $96^{a}$ & 20 & 4,8 & 68 \\
\hline Michelson (1991) & 13 & 19 & 56 & 81 & 0 & 0 & $69^{a *}$ & 17 & 4,1 & 101 \\
\hline Shearer (1987) & 77 & 60 & 0 & 0 & 52 & 40 & $129 * a$ & 32 & 4,0 & 120 \\
\hline Tisserand (2004) & 31 & 32 & 47 & 48 & 19 & 20 & $97^{*}$ & 19 & 4,9 & 48 \\
\hline Total des 10 méthodes & 543 & & 277 & & 211 & & 1031 & & & \\
\hline
\end{tabular}

a les accompagnements destinés à être joués par l'enseignant et qui sont écrits directement dans la méthode.

* les méthodes dont les accompagnements sont fournis sur disque compact

Dans les méthodes, toutes proportions gardées, le nombre d'exercices présents est plus élevé que le nombre de mélodies et de pièces réunies, sauf chez Michelson (1991) et Tisserand (2004) où les mélodies prédominent. Nous

13 Un grand barré implique que l'index de la main gauche appui fortement sur les six cordes simultanément, bien souvent en même temps que d'autres doigts qui sont aussi placés sur les cordes. On comprend que cela demande de la force, du contrôle et de la dextérité. 
constatons que cinq méthodes proposent entre 4 à 4,9 items pour travailler un nouvel élément d'apprentissage. Benoît (1991) propose seulement 3,6 activités. À l'opposé du spectre, McKinnon1 et Koyama (1975) en proposent respectivement 6 et 6,4, alors que Camisassa (2002) et Lemay (1990) offrent plus de 8 items pour travailler chacun des éléments d'apprentissage proposés.

Dans le tableau 4, les astérisques indiquent les méthodes dont les accompagnements sont fournis sur disque compact et les " $^{\mathrm{a}}$ » indiquent les accompagnements destinés à être joués par l'enseignant et qui sont écrits directement dans la méthode. Tisserand (2004) est le seul qui ne prévoit pas d'accompagnement écrit dans la méthode. Cependant, tout comme Michelson (1991), Duncan (1996) et Shearer (1987), un disque compact d'accompagnement est fourni avec la méthode. Benoît (1991), Duncan (1996), Lemay (1990) McKinnon1, McKinnon2 et Shearer (1987) ont écrit tous les accompagnements à la note, alors que Michelson (1991) inscrit seulement les lettres représentant les accords à jouer. Selon le répertoire, certains auteurs comme Camisassa (2002) utilisent les deux types de notation. Il est à noter que Shearer (1987) offre un petit logiciel permettant de ralentir les accompagnements fournis sur le disque. Seul Tisserand (2004) n'offre qu'un disque d'accompagnement sans autre matériel d'accompagnement.

\section{Indice densité et nombre de pages}

Un aspect surprenant à première vue est la grande disparité entre les méthodes quant aux éléments d'apprentissage ${ }^{14}$ proposés en regard du nombre de pages. Par exemple, Michelson (1991) propose 17 éléments d'apprentissage dans une méthode qui contient 101 pages alors que Tisserand (2004) proposent 19 éléments d'apprentissage dans une méthode de 48 pages (Tableau 4). Plusieurs facteurs expliquent cette disparité, dont la mise en pages. En effet, Michelson (1991) propose souvent un seul exercice ou une seule monodie par page, y intégrant quelques explications, des pages de présentation pour chacune des leçons proposées, des photos d'enfants illustrant les exercices, et plusieurs recommandations et idées pédagogiques adressées à l'enseignant ou au parent. $\mathrm{Au}$ contraire, Tisserand (2004), qui s'adresse directement à l'enfant, propose jusqu'à quatre courtes mélodies pour un élément d'apprentissage.

Cependant, les indices de densité nous informent sur la cohérence des choix et des séquences pédagogiques et nous permettent de distinguer trois groupes de méthodes: celles dont l'indice est de 6 ou plus, celles dont l'indice est inférieur à 4, et les autres, dont l'indice est entre 4 et 4,9. Plus les éléments d'apprentissage sont complexes dans une méthode, plus les indices de densité sont élevés, et plus il y a d'activités proposées afin d'en permettre l'assimilation. Par exemple, la méthode de Koyama (1975), qui présente un indice de 6,4, soit beaucoup d'activités par éléments d'apprentissage, présente aussi les éléments d'apprentissage les plus denses de notre corpus (notes simultanées, introduction rapide des techniques de jeu polyphonique), ce qui justifie le nombre élevé

14 Rappelons qu'un élément d'apprentissage est constitué de notions et d'habileté à faire acquérir, bien identifié par les auteurs, accompagné d'une séquence d'apprentissage qui comprend des explications, des exercices ou des œuvres musicales. 
d'exercices, de mélodies ou de pièces pour permettre leur assimilation. Il en est de même pour Camisassa (2002), dont l'indice est de 8,1 pour 16 éléments d'apprentissage qui, de plus, propose vers la fin de son volume beaucoup de pièces permettant d'assimiler les notions et habiletés présentées. Par opposition, si les éléments d'apprentissage sont simples, un moins grand nombre d'exercices est nécessaire à leur assimilation, ce que nous constatons dans la majorité des méthodes: Duncan (1996), McKinnon, 2, Michelson (1991), Shearer (1987) et Tisserand (2004). Nous voyons cependant deux exceptions: Benoît (1991) qui propose huit éléments d’apprentissage simples et Lemay (1990) qui en propose 18, comprenant souvent de nouvelles notes et des rythmes introduits de manière individuelle et graduelle. L'assimilation de ces nouveaux éléments est soutenue par plusieurs activités, notamment sous forme de monodies. Nous pouvons ainsi conclure que la méthode de Lemay (1990) est la plus graduelle puisqu'elle offre davantage d'activités d'apprentissage afin de permettre l'assimilation des notions et des habiletés.

\section{Provenance du répertoire}

Le tableau présente la provenance du répertoire (mélodies et pièces) que l'on retrouve dans les méthodes de notre corpus. Nous observons une prévalence du répertoire composé par l'auteur dans l'ensemble du corpus, même si cette catégorie est absente chez Koyama (1975). Le pourcentage du répertoire composé par l'auteur est majoritaire chez les auteurs suivants : $87 \mathrm{chez} \mathrm{McKinnon} \mathrm{1,}$ 86 chez Camisassa (2002), 83 chez Tisserand (2004), 72 chez McKinnon 2 et $69 \mathrm{chez}$ Shearer (1987). Le folklore est la deuxième catégorie la plus populaire. Elle prédomine chez Michelson (1991) avec 82, chez Duncan (1996) avec 71, chez Benoit (1977) avec 62 et chez Lemay (1990) avec 59. Le répertoire du $19^{\mathrm{e}}$ siècle est aussi bien représenté: à 27 chez Shearer et majoritaire chez Koyama (1975) à 68, en grande partie parce que ces deux méthodes reprennent du répertoire polyphonique de compositeurs guitaristes célèbres du début du XIX ${ }^{\mathrm{e}}$ siècle, notamment Sor, Carulli et Carcassi. Nous pouvons également constater que les XVI ${ }^{e}$, $\mathrm{XVII}{ }^{\mathrm{e}}$ et XVIII ${ }^{\mathrm{e}}$ siècles sont peu présents dans les méthodes.

Tableau 5. Provenance du répertoire

\begin{tabular}{|c|c|c|c|c|c|c|c|c|c|c|c|c|c|c|}
\hline & & 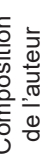 & & 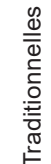 & & 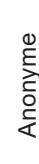 & & $\begin{array}{l}\frac{0}{0} \\
: \frac{\mathbb{d}}{\omega} \\
0 \\
\stackrel{0}{N}\end{array}$ & & 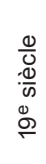 & & $\begin{array}{l}\frac{0}{U} \\
: \frac{1}{\omega} \\
\infty \\
\infty\end{array}$ & & 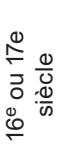 \\
\hline & $\mathrm{N}^{\text {bre }}$ & $\%$ & $\mathrm{~N}^{\text {bre }}$ & $\%$ & $\mathrm{~N}^{\text {bre }}$ & $\%$ & $\mathrm{~N}^{\text {bre }}$ & $\%$ & $\mathrm{~N}^{\text {bre }}$ & $\%$ & $\mathrm{~N}^{\text {bre }}$ & $\%$ & $\mathrm{~N}^{\text {bre }}$ & $\%$ \\
\hline Benoît (1991) & 11 & 38 & 18 & 62 & & & & & & & & & & \\
\hline Camisassa (2002) & 50 & 86 & 6 & 10 & & & & & 1 & 2 & & & 1 & 2 \\
\hline Duncan (1996) & 14 & 29 & 30 & 63 & & & & & 4 & 8 & & & & \\
\hline Koyama (1975) & & & 13 & 27 & & & & & 33 & 67 & 2 & 4 & 1 & 2 \\
\hline
\end{tabular}




\begin{tabular}{|c|c|c|c|c|c|c|c|c|c|c|c|c|c|c|}
\hline & & 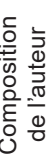 & & 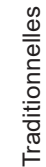 & & है & & 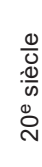 & & $\begin{array}{l}\frac{0}{0} \\
: \frac{\mathbb{d}}{\omega} \\
\Phi \\
\frac{\sigma}{\Gamma}\end{array}$ & & 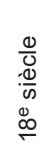 & & 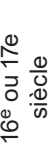 \\
\hline & $N^{\text {bre }}$ & $\%$ & $\mathrm{~N}^{\text {bre }}$ & $\%$ & $N^{b r e}$ & $\%$ & $N^{\text {bre }}$ & $\%$ & $N^{\text {bre }}$ & $\%$ & $\mathrm{~N}^{\text {bre }}$ & $\%$ & $\mathrm{~N}^{\text {bre }}$ & $\%$ \\
\hline Lemay (1990) & 14 & 23 & 36 & 59 & 3 & 5 & 6 & 10 & 1 & 2 & 1 & 2 & & \\
\hline $\begin{array}{l}\text { McKinnon, } 1 \\
\text { (1984) }\end{array}$ & 13 & 87 & 2 & 13 & & & & & & & & & & \\
\hline $\begin{array}{l}\text { McKinnon, } 2 \\
(2004)\end{array}$ & 31 & 72 & 5 & 12 & & & & & 5 & 12 & 2 & 5 & & \\
\hline Michelson (1991) & 4 & 7 & 46 & 82 & 2 & 4 & 1 & 2 & & & 3 & 5 & & \\
\hline Shearer (1987) & 36 & 69 & 1 & 2 & 1 & 2 & & & 14 & 27 & & & & \\
\hline Tisserand (2004) & 55 & 83 & 9 & 14 & & & & & 2 & 3 & & & & \\
\hline AU TOTAL & & 46 & & 36 & & 2 & & 12 & & & & 16 & & \\
\hline
\end{tabular}

\section{Aspects qualitatifs du jeu}

Shearer (1987) et Michelson (1991) mentionnent l'importance de jouer à un tempo confortable afin de s'assurer d'un jeu de qualité. La musicalité est abordée par Shearer (1987) et Michelson (1991) qui mentionnent la nécessité de la développer autant que la technique. Lemay (1990), Michelson (1991), Shearer (1987) et Tisserand (2004) mentionnent qu'il est important de porter attention à l'enchaînement des sons pour que le legato soit maintenu. Les nuances sont indiquées pour deux morceaux de la méthode de Benoît (1991) (des crescendo et decrescendo), six de la méthode de Camisassa (2002), sept chez Koyama (1975) et six chez Tisserand (2004). Ce dernier s'intéresse au phrasé en indiquant les phrases musicales grâce à une explication sur le besoin de respirer dans la musique et à l'aide de virgules dans neuf pièces. Duncan (1996) offre une simple explication sur la structure de la pièce Au Clair de la lune.

Les activités dans lesquelles la création des apprentis guitaristes est mise à contribution sont rares. En effet, seules deux méthodes proposent, de manière très ponctuelle, des activités de création. Benoît (1991) propose, au début de sa méthode, l'apprentissage d'une pièce monodique sur trois notes (sol, la, si), qui comporte un refrain et trois couplets. Il ajoute: «Lorsque les exécutants pourront le jouer avec facilité, ils pourront composer leurs propres couplets» (p. 9). Quant à Tisserand (2004), il propose une page remplie de portées vides avec les consignes suivantes: «Tu peux utiliser cette page pour écrire un air connu dont tu as trouvé les notes», «... ou ton professeur peut écrire des exercices si tu en as besoin», « ... ou écrire des morceaux que tu as inventés ...» (p. 31). 


\section{Notions théoriques}

Des éléments de théorie musicale sont présentés dans huit ouvrages sur dix. Peu élaborés, ils permettent simplement l'acquisition des notions de base essentielles à la lecture de la partition: explications au sujet de la portée, du nom des notes, des figures rythmiques et de silence, des indicatifs de mesure et de la répartition des temps dans une mesure. Ainsi, seul Benoît (1991) et Michelson (1991) ne présentent pas de notions théoriques. Par contre, presque tous les ouvrages spécifient les symboles typiques au jeu de la guitare que l'on retrouve sur la partition (par exemple, le système pour identifier les cordes).

Camisassa (2002) et Michelson (1991) sont les seuls auteurs à ne pas présenter un schéma, un dessin ou une photo illustrant les parties de la guitare. Michelson (1991) est le seul auteur qui n'explique pas les symboles utilisés pour désigner les doigtés. Camisassa (2002), McKinnon 1 et 2, Michelson (1991) et Tisserand (2004) n'indiquent pas de moyens pour accorder la guitare.

Sur les dix ouvrages analysés, certains auteurs présentent des stratégies d'apprentissage ou d'enseignement souvent dans l'introduction de leur méthode. Ainsi, sur dix ouvrages analysés, quatre auteurs offrent des stratégies de travail. Tisserand (2004) propose un tableau dans lequel l'élève ou le professeur peut écrire ce qui est à travailler pour le prochain cours. Duncan (1996) propose cinq consignes générales simples pour les élèves. Voici un exemple : "Play at least half-hour a day on as regular a schedule as possible» (p. 3$)^{15}$. Shearer (1987) est l'auteur qui offre le plus de stratégies et de consignes pour le travail. Il y consacre une page entière, dans laquelle il propose entre autres de travailler lentement en petites sections pour minimiser les erreurs et propose même de visualiser: "Finally, visualize and recite the two segments together and then play them» (p. 26) ${ }^{16}$. Michelson (1991) offre des principes pour favoriser le travail instrumental, mais qui s'adressent aux parents et aux enseignants. Rappelons que la méthode s'adresse à des enfants de trois ans et plus selon l'auteur, et que des consignes d'enseignement sont offertes tout au long de l'ouvrage. Par exemple, l'auteur suggère ceci: «Have the student listen to his or her own playing by taping the piece during the lesson» (p. 54) ${ }^{17}$. Une section de cinq pages en début de méthode est consacrée aux consignes destinées aux enseignants et présente des exercices d'éveil musical.

\section{Discussion}

L'identification de ces principes pédagogiques pourrait permettre une réflexion approfondie sur la pédagogie instrumentale ainsi qu'une remise en question des pratiques traditionnelles à la lumière des récentes recherches. Notre hypothèse de départ était qu'un ensemble de croyances, de règles et de valeurs agit comme cadre de référence et conditionne les choix pédagogiques des musiciens enseignants selon la tradition dont ils sont issus. Nous avons analysé des

15 Traduction libre : Jouez au moins une demi-heure par jour et selon un horaire régulier.

16 Traduction libre : Finalement, visualiser et solfier les deux segments ensemble, puis les jouer.

17 Traduction libre : faites en sorte que l'étudiant puisse entendre son jeu en l'enregistrant durant le cours. 
méthodes de guitares destinées aux débutants suggérées par les organismes d'encadrement de l'enseignement musical en privé. Voici les paradigmes que nous avons identifiés.

\section{Le but des méthodes}

Un premier paradigme est commun à toutes les méthodes analysées et touche leur raison d'être: les méthodes sont conçues pour favoriser l'acquisition des habiletés instrumentales et des connaissances théoriques permettant de jouer un répertoire d'œuvres écrites. En effet, tout comme dans l'étude de Sundell (2012) au sujet des méthodes de piano publiées en Amérique du Nord, nous constatons que les méthodes de notre corpus se concentrent sur le jeu instrumental et la lecture des notes. La théorie musicale est limitée aux notions de base essentielles au déchiffrage de la musique et les activités de création sont à peu près inexistantes. Dans le corpus qu'elle a étudié, Sodoyer (1995, p. 118) constate l'apparition d'activités qui demandent d'improviser dès 1983 dans 18 méthodes, mais ce type d'activité est pratiquement absent dans les ouvrages que nous avons analysés. Par contre, la présence de parties d'accompagnement observées chez Sodoyer dès 1989 est une pratique devenue courante, car tous les ouvrages analysés en proposent, qu'elles soient écrites dans la méthode ou présentes sur un disque, ou les deux. Nous pouvons inférer que cela favorise la motivation des élèves, un peu comme l'invoquait Sundell (2012) au sujet du répertoire en duo présent dans les ouvrages qu'elle a analysés.

De plus, les éléments liés au concept de comprehensive musicianship tels que définis chez Sundell (2012) sont rares, sauf chez deux auteurs Michelson (1991) et Shearer (1987). Michelson (1991) propose beaucoup de stratégies de travail et d'activités de développement musical général (entre autres, la formation de l'oreille), mais son ouvrage s'adresse ouvertement aux enseignants et aux parents. Shearer (1987), quant à lui, est le seul qui propose des sections spécifiques consacrées à l'achat d'un instrument, aux stratégies de travail, à l'interprétation et à la connaissance des compositeurs pour guitare.

\section{Les techniques de jeu proposées sont simples}

Le deuxième paradigme est commun à toute les méthodes, sauf celle de Koyama (1975): les techniques de jeux présentées dans les méthodes analysées, qui sont majoritairement destinés aux jeunes apprenants, sont simples, à l'image de ce qu'avait constaté Sodoyer (1995, p. 116) dans les ouvrages publiés après 1970. Il faut noter que Koyama (1975) nous offre un ouvrage assez atypique: les techniques de jeu proposées sont complexes (introduction de changements de positions, de liés, de barrés), plusieurs notes sont introduites dans un même élément d'apprentissage, le répertoire est presque exclusivement polyphonique avec l'utilisation des notes présentes sur les cordes basses fermées et les rythmes sont plus complexes, même dans les œuvres polyphoniques.

Le troisième paradigme touche la séquence d'apprentissage des notes: de manière générale, les notes sont introduites graduellement et avec des rythmes simples. Les gammes ne structurent pas les ouvrages en présentant 
un ensemble de notes à assimiler simultanément, comme c'était le cas avant les années 1970 (Sodoyer 1995, p. 70). D’ailleurs, l'ajout graduel d'une note à la fois est adopté par Benoît (1991), Lemay (1990), Michelson (1991), McKinnon 1 (1984) et McKinnon 2 (1984). L'introduction de plusieurs notes qui figurent sur une même corde est préconisé par Duncan (1996) McKinnon 2, Shearer (1987) et Camisassa (2002).

Si ce paradigme de l'introduction graduelle des notes est partagé, l'ordre d'apparition des notes est réalisé selon deux approches: l'apprentissage des notes sur les cordes ouvertes ou celui sur les cordes fermées, avec des impacts différents. Par exemple, les auteurs qui choisissent d'introduire les notes en cordes ouvertes dès le début des apprentissages permettent au débutant de porter son attention sur une seule main et de se concentrer sur l'attaque de la corde. Les pièces et exercices proposés offrent des monodies avec les notes disjointes $m i$, si, et sol, comme chez Tisserand (2004, p. 9) et favorisent rapidement l'introduction de la polyphonie grâce à l'usage du pouce sur les cordes basses ré, la et $m i$, par exemple chez Shearer (1987, p. 32).

Les auteurs qui préconisent la deuxième approche, Benoît (1991), Koyama (1975), Lemay (1990) et Michelson (1991) introduisent rapidement l'action de la main gauche pour changer la hauteur des sons, ce qui demande une synchronisation entre les deux mains dès le début du jeu. Ceci permet le jeu de mélodies constituées de notes conjointes et favorise le jeu d'airs connus. D’ailleurs, ces mêmes auteurs introduisent la lecture de notes dès le tout début du jeu avec des rondes, des blanches ou des noires et le répertoire abordé est monodique ${ }^{18}$. Souvent, l'introduction de nouvelles notes et de nouveaux rythmes est rapide, surtout chez Lemay (1990), ce qui permet une variété dans le répertoire, mais pourrait constituer un défi psychomoteur dû à la combinaison des exigences de lecture avec celles des gestes exigés pour la synchronisation des deux mains. Nous pourrions conclure que ces derniers auteurs favorisent le jeu d'un riche répertoire de monodies, souvent connues, alors que les premiers, avec l'utilisation des cordes ouvertes, favorisent plutôt l'acquisition des mouvements permettant plus rapidement le jeu d'un répertoire polyphonique.

\section{Le répertoire doit être adapté à l'apprentissage projeté}

Le troisième paradigme concerne le répertoire proposé aux jeunes débutants: il est simple, majoritairement composé par l'auteur ou alors de provenance folklorique. Tout comme dans l'étude de $\mathrm{Lu}$ (2012), le répertoire des méthodes que nous avons analysées est majoritairement constitué d'œuvres composées par les auteurs, en proportion différente cependant, et souvent sous forme de pastiche et de pièces folkloriques ne laissant que peu de place au répertoire plus ancien. On peut expliquer le fait que les auteurs composent eux-mêmes le répertoire de leur méthode par la volonté d'en contrôler le niveau de difficulté et de l'adapter aux apprentissages projetés. Par exemple, ils peuvent choisir les notes et les techniques de jeu utilisées en lien avec la progression des éléments d'apprentissage de leur ouvrage.

18 Notons que Koyama (1975) introduit aussi rapidement le jeu polyphonique. 
Koyama (1975) fait figure d'exception avec un répertoire à 68 constitué d'œuvres du XIX ${ }^{\mathrm{e}}$ siècle et à 26 de pièces traditionnelles, de quelques pièces d'autres époques, mais d'aucune composition originale. D'ailleurs, le répertoire proposé comporte de nombreux défis de technique et de lecture avec une progression très rapide.

\section{Conception des éléments apprentissages et indice de densité}

Le quatrième paradigme est le suivant: plus un élément d'apprentissage est complexe, plus le nombre d'activités proposées à l'apprenant est élevé. Il a été surprenant de constater que cet élément d'apprentissage varie énormément d'un auteur à l'autre. Par exemple, chez Tisserand (2004), les notes sur les trois premières cordes ouvertes, $\mathrm{mi}$, si et sol, sont introduites individuellement, avec une mélodie consacrée à chacune, comme autant d'éléments d'apprentissage différents (p. 7). Pour Koyama (1975), qui s'adresse à des enfants du même âge, le jeu des six cordes ouvertes constitue un seul élément d'apprentissage qui est accompagné de deux exercices qui les regroupent (p. 6). D’ailleurs, cet auteur regroupe en un seul élément d'apprentissage des notions ou habiletés qui figurent comme autant d'éléments différents chez d'autres auteurs. La progression proposée est rapide, un peu à l'image des anciennes méthodes analysées par Sodoyer (1995).

La conception de ce qui constitue un élément d'apprentissage a un impact sur la densité des activités proposées. En effet, plus les éléments d’apprentissage sont complexes dans une méthode, plus les indices de densité sont élevés, et plus d'activités sont proposées afin d'en permettre l'assimilation. Par exemple, la méthode de Koyama (1975), qui offre un indice de 6,4 - soit beaucoup d'activités par éléments d'apprentissage —, présente aussi les éléments d’apprentissage les plus denses de notre corpus (notes simultanées, introduction rapide des techniques de jeu polyphonique), ce qui justifie le nombre élevé d'exercices, de mélodies ou de pièces pour permettre leur assimilation. Il en est de même pour Camisassa (2002), dont l'indice est de 8,1 pour 16 éléments d'apprentissage qui, en plus, propose beaucoup de pièces permettant d'assimiler les notions et habiletés présentées vers la fin de son volume. Par opposition, si les éléments d'apprentissage sont simples, un moins grand nombre d'exercices est nécessaire à leur assimilation, ce que nous constatons chez Duncan (1996), McKinnon 2 (1984), Michelson (1991), Shearer (1987) et Tisserand (2004). Nous voyons cependant deux exceptions: Benoît (1991) qui propose huit éléments d'apprentissage simples et Lemay (1990) qui en propose 18, comprenant souvent de nouvelles notes et des rythmes introduits de manière individuelle et graduelle. L'assimilation de ces nouveaux éléments est soutenue par plusieurs activités, notamment sous forme de monodies. Nous pouvons ainsi conclure que la méthode de Lemay (1990), et dans une moindre de mesure celle de Benoit, sont les plus graduelles puisqu'elles offrent davantage d'activités d'apprentissage afin de permettre l'assimilation des notions et des habiletés. 


\section{Impact de l'âge visé}

Le cinquième paradigme est à l'effet que l'âge des apprenants visés par les méthodes a un impact sur le contenu des méthodes et sur leur présentation. Les méthodes s'adressant aux plus jeunes, soit de trois à six ans, présentent des polices de caractère plus grosses, des dessins fantaisistes, de la couleur, ou des photos d'enfants représentant l'âge ciblé Tisserand (2004) Michelson (1991) McKinnon 1 (1984). De plus, ces méthodes proposent exclusivement le jeu monodique, sauf pour Koyama (1975).

Les méthodes qui s'adressent aux débutants dès sept ou huit ans, McKinnon 2 (2004), Duncan (1996), Benoît (1991) et Lemay (1990), présentent déjà une mise en pages plus adulte et introduisent le jeu polyphonique dans la dernière partie de leur ouvrage.

Camisassa (2002) semble d'adresser aux apprenants de 10 ans et plus, comme Shearer (1987) qui lui s'adresse aussi à un public plus âgé (photo d'adultes). Ces deux ouvrages proposent plus d'explications et introduisent le jeu polyphonique dès le début des apprentissages.

\section{Deux ouvrages atypiques}

Il est intéressant de constater que bien que l'ouvrage de Shearer (1987) soit le plus ancien de notre corpus (la première édition de cette méthode date de 1959), il est conforme aux observations de Sodoyer (1995) à certains égards. En effet, les méthodes les plus anciennes s'adressent aux apprentis adultes, abordent principalement le jeu polyphonique, avec du répertoire composé par l'auteur et proposent un chapitre à la fin de leur méthode sur l'interprétation et les compositeurs à connaître (p. 106-108). Dans l'ouvrage de Shearer (1987), une grande partie du répertoire est composé par l'auteur, et une section, à la fin de la méthode, présente des œuvres des principaux compositeurs de l'époque romantique, mais aussi des conseils sur l'interprétation musicale, les principaux compositeurs à connaître et à interpréter. Notons cependant que les techniques de jeu utilisées sont simples, et que l'apprentissage des notes se fait de manière graduelle, contrairement aux méthodes ancienne (Sodoyer 1995).

Bien que la méthode Koyama (1975) ait été éditée plus tardivement, elle adopte certaines caractéristiques des méthodes anciennes et présente des différences flagrantes avec l'ensemble de notre corpus. Bien qu'elle s'adresse aux jeunes enfants, la progression est rapide, le répertoire est polyphonique, tiré principalement du XIX ${ }^{\mathrm{e}}$ siècle et les défis techniques et de lecture sont nombreux. Si l'auteur se réclame de l'approche Suzuki dans l'introduction de son ouvrage, la conception de celui-ci semble reposer sur d'anciens paradigmes pédagogiques.

\section{LIMITES ET CONCLUSION}

Cette étude visait à développer une méthodologie pour analyser le matériel éducatif utilisé dans l'enseignement de la guitare classique afin d'en dégager des paradigmes pédagogiques, ce qui n’avait jamais été réalisé auparavant. Les pédagogues pourront s'y référer, pour réfléchir à leur conception de 
l'enseignement et de l'apprentissage de la guitare et pour choisir leur recherche de matériel pédagogique. Par contre, si certaines retombées sont pragmatiques, certaines sont aussi d'ordre méthodologique ou didactique, et soulèvent plus de questions qu'elles n'offrent de réponses.

Les données concernant le contenu des méthodes, leur mise en forme et leur traitement avec le logiciel Excel constituent une banque de données riche qui n’a pas fini de révéler la manière dont l'enseignement de la guitare est conçu. Les données nous informent sur des éléments très pointus, comme la manière d'aborder les changements de cordes dans l'apprentissage des cinq premières notes, qui, s'ils sont d'un intérêt certain pour la pédagogie de la guitare, ne peuvent être tous exposés ici. On conçoit aisément qu'une telle base de données peut aussi s'enrichir chaque année des nouvelles publications et être interrogée pour étudier plusieurs aspects didactiques.

Il faut cependant reconnaître que l'analyse du contenu des méthodes ne permet pas de prendre en compte tous les aspects qui caractérisent l'enseignement de l'instrument. En effet, elle n'indique pas comment le musicien pédagogue utilise le matériel pédagogique: est-ce qu'il l'enrichit de différents exercices ou est-ce qu'il omet certaines parties? De plus, les musiciens pédagogues développent souvent eux-mêmes du matériel pédagogique qu'ils utilisent dans les cours afin de répondre aux spécificités de leurs élèves (Héroux 2006, p. 16). Pour avoir une vision plus exhaustive du matériel pédagogique, il serait donc pertinent d'analyser le matériel produit et non publié, mais effectivement utilisé dans les cours d'instruments. De plus, il serait important d'analyser les pratiques pédagogiques in vivo pour enrichir notre compréhension des paradigmes pédagogiques de l'enseignement de la guitare aux jeunes débutants. En effet, cette analyse du matériel utilisé ne nous donne pas accès à la pratique pédagogique du musicien pédagogue, à savoir s'il utilise le modelage ou s'il favorise l'expérimentation.

Néanmoins, l'analyse des méthodes nous permet d'identifier les éléments qui se sont frayé une place dans les ouvrages, donnant ainsi des indices de ce qui est valorisé par les auteurs. Nous devons constater que l'apprentissage des notes à l'instrument et leur lecture sur la partition grâce à un répertoire d'exercices et de pièces à reproduire occupent pratiquement toute la place. Par contre, le chemin emprunté par les auteurs est différent, comme nous l'avons constaté avec la manière d'aborder ou non le jeu monodique ou polyphonique, le choix de présenter les notes sur les cordes ouvertes avant les notes sur les cordes fermées ou l'inverse. Ces décisions pédagogiques reposent sur des conceptions de l'enseignement de la guitare, voire même de la nature de l'instrument. Par exemple, privilégier le jeu sur les cordes ouvertes permet l'apprentissage des techniques d'attaque à la main droite et peut favoriser le jeu polyphonique avant le travail du synchronisme entre les deux mains et l'acquisition d'un grand répertoire de notes. Par contre, introduire dès le départ la synchronisation entre les deux mains pour changer la hauteur des sons, ce qui peut paraître plus complexe d'un point de vue cognitif et moteur, permet une variété plus grande dans les pièces jouées, souvent monodiques et connues de l'élève. Il nous faudrait expérimenter ces deux méthodes afin de savoir laquelle est la 
plus facile pour les débutants, la plus motivante selon l'âge des apprenants. Par contre, nous pouvons nous interroger: à notre connaissance, rares sont les pédagogues qui proposent aux pianistes débutants d'apprendre un répertoire musical essentiellement monodique pendant plusieurs mois.

Différents aspects sont pratiquement absents des méthodes analysées. Par exemple, l'approche globale de la musique, avec le développement de la créativité, est pratiquement absente. Ceci est étonnant, car la création et l'appréciation sont au cœur des programmes d'enseignement de la musique en milieu scolaire au Québec $^{19}$. Les éléments qui ont trait à l'expressivité du jeu, aux nuances, aux accents, aux phrasés et aux timbres sont aussi peu présents, bien qu'essentiels à la musique. Ainsi, il nous est impossible de savoir à quel moment de l'apprentissage de la guitare la grande majorité des auteurs dont les méthodes ont été analysées considèrent qu'un élève peut travailler l'expressivité. Ce résultat n'est cependant pas surprenant. En effet, Juslin \& Persson (2002, p. 220) ont déjà fait état d'études démontrant que les professeurs de musique consacrent plus de temps à enseigner la technique instrumentale que l'expressivité et l'interprétation. De plus, les exercices préparatoires au jeu instrumental (sans guitare) ainsi que les exercices d'étirement après une séance de répétition sont inexistants. On peut invoquer le fait que les apprentissages d'un débutant ne sollicitent pas le corps à un point tel que les auteurs doivent s'assurer de prévenir les blessures dans leur méthode. Nous pouvons aussi penser que cette partie de l'enseignement est laissée à la discrétion des pédagogues en présence des élèves. Pourtant, certains pédagogues proposent d'intégrer des exercices pour aider à l'échauffement et à la détente, favorisant ainsi une plus grande conscience corporelle dès le plus jeune âge (Vuillemin 1997, p. 83).

Nous pourrions conclure que la présence ou l'absence de ces éléments dans la planification de ce qui doit être enseigné aux débutants est laissée à la discrétion des enseignants, mais aussi qu'elle reflète une conception conservatrice de l'enseignement de la musique dans laquelle l'objectif de reproduire fidèlement la partition domine toujours. Si les musiciens pédagogues ont tendance à reproduire les enseignements reçus et à utiliser un matériau éducatif qui reflète leur conception de l'apprentissage de la guitare, la lecture d'une analyse de contenu de différentes méthodes telles que celle-ci pourrait permettre une ouverture sur ce qui peut être conçu et enseigné autrement, et, qui sait, favoriser une saine interrogation sur ses propres pratiques.

\section{RÉFÉRENCES}

Albergo, C., F. 1988. «Objectives for elementary level piano instruction: A survey and comparison of the objectives of eight American children's piano methods with the objectives of piano/piano pedagogy teachers». Thèse de doctorat, Music Faculty, University of Illinois.

Albergo, C., F. 2003. Celebrate Piano! sous la dir. de M. Kolar. Ontario : Frederick Harris Music CO Limited.

Bardin, Laurence. 2003. L'analyse de contenu. 10 éd, Quadrige Manuels. Paris: PUF. 
Bastien, James W. 1998. Méthode de piano Bastien. Niveau 1, sous la dir. de Jane Smisor Bastien. San Diego, Calif.: Fontenay sous Bois, France: San Diego, Calif.: Neil A. Kjos Music Company ; Fontenay sous Bois, France: MUSICom.

Benoit, Florence. 1995. "Évolution des Méthodes de guitare au XXe siècle.» Mémoire de maîtrise, Université de Paris-Sorbonne.

Benoit, Marcel. 1991. Méthode de guitare. Richelieu: Musantiqua.

Bruneau, Monik, and Sophia L. Burns. 1990. «La recherche création. Entre désir, paradoxes et réalités de la recherche.», Traiter de recherche création en art: entre la quête d'un territoire et la singularité des parcours, sous la dir. de Monik Bruneau et André Villeneuve, 7-20. Sainte-Foy: Sainte-Foy: Presses de l'Université du Québec.

Camisassa, Claudio. 2001. "Méthode progressive : guitare = Progressive method : guitar = Método progresivo : guitarra ", Progressive method. SaintRomuald: D'Oz.

Delcamp, Jean-François. 1984. Un éventail des méthodes de guitare (12).

Duncan, Charles. 1996. Modern Approach to Classical Guitar. Milwaukee : Hal Leonard.

Faber, N. 1996. Piano Adventure, sous la dir. de R. Faber. Miami Beach : The FJH Music company Inc.

Favre, Georges. 1988. Histoire de l'éducation musicale. Montréal: Montréal Livrophile.

Gauthier, Clermont. 2012. "Le XVII ${ }^{\mathrm{e}}$ siècle et la naissance de la pédagogie». La pédagogie: théories et pratiques de l'Antiquité à nos jours, sous la dir. de Clermont Gauthier et Maurice Tardif, 59-74. Montréal: G. Morin.

Héroux, Isabelle. 2006. «L'apport de la psychologie cognitive dans l'élaboration d'un outil didactique pour favoriser l'apprentissage de la guitare au niveau collégial.» Thèse de doctorat, Université Laval (1).

-2008. À vos guitares, prêts? Partez! Édité par didactique Centre collégial de développement de matériel. Montréal: CCDMD.

- 2009. "Processus méthodologique pour le développement de matériel didactique.» L'éducation musicale au $\mathrm{XXI}^{\mathrm{e}}$ siècle: Quelle recherche? Quelle formation? Journée francophone de recherche en musique, Ottawa. Juslin, Patrick, N., and Roland Persson, S. 2002. «Emotional Communication», The Science and Psychology of Music Performance», sous la dir. de Richard Parncutt et Gary McPherson, 219-236. New-York: Oxford University Press. Koyama, Masaru. 1975. Guitar Method for Children. Tokyo : Zen-On Music. L'Écuyer, René. 1990. Méthodologie de l'analyse développementale de contenu méthode GPS et concept de soi. Sillery: Sillery Presses de l'Université du Québec.

Lartigot, Jean-Claude. 1999. L'apprenti instrumentiste [de la logique des méthodes à la médiation des professeurs dans la situation de l'enseignement] de la logique des méthodes à la médiation des professeurs dans la situation de «enseignement, Collections: Musique et société. Tours: Van de Velde.

Legendre, Rénald. 2005. "Dictionnaire actuel de l'éducation", Dictionnaire actuel de l'éducation, sous la dir. de Rénald Legendre. Montréal: Guérin. 
Lemay, Sylvain. 1990. Méthode de guitare pour débutants. Sillery: Éditions d'OZ.

Lescat, Philippe. 1991. Méthodes et traités musicaux en France, 1660-1800: réflexions sur l'écriture de la pédagogie musicale en France, suivies de catalogues systématique et chronologique, de repères biographiques et bibliographiques, Méthodes \& traités musicaux en France, 1660-180o. Paris: Institut de pédagogie musicale et chorégraphique.

Lu, Yuanyuan. 2012. "Survey of Eighteen North - American Piano Method Books: Repertoire Selection and Categories ». Mémoire de maîtrise, Université d'Ottawa.

McKinnon, Claude. 2004. Mes premiers pas à la guitare. Charlesbourg: Éditions Geneviève.

—_.1984. J'apprivoise la guitare. Charlesbourg: Éditions Geneviève.

Membrado, Florence. 1983. Ma première année de guitare. Paris: Alphonse Leduc.

Michelsson, Sonia. 1991. New dimension in Classical : Guitar for Children. Pacific : Mel Bay.

Ribouillot, Danièle. 1980. «La technique de guitare en France dans la première moitié du XIXe siècle.» Thèse de doctorat, Université Paris 4.

Shearer, Aaron. 1987. Classic guitar technique. $3^{\text {rd }}$ ed. rev., Concert guitar technique. New York: New York F. Colombo.

Sundell, Kimberley. 2012. "Comprehensive Musicianship and Beginner Piano Method Books: A Content Analysis». Mémoire de maîtrise, University of Ottawa.

Tisserand, Thierry. 2004. «Je deviens guitariste, vol. 1, Méthode pour tous les débutants en $1^{\mathrm{re}}$ année», Méthode pour tous les débutants en $1^{\text {re }}$ année. Paris: Paris: H. Lemoine.

Valois, Pascal. 2003. «Les guitaristes français entre 1770 et 1830: Pratiques d'exécution et catalogue des méthodes». Thèse de doctorat, Université Laval.

Vuillemin, Dominique. 1997. L’apprentissage de la musique guide à l'usage des parents. Paris: Paris Éditions du Seuil. 


\section{Annexe 1}

Extrait feuille de calcul Excel

\begin{tabular}{|c|c|c|c|c|c|c|c|c|c|c|}
\hline Ordre & & $\begin{array}{c}0 \\
\text { (intro) }\end{array}$ & 1 & 2 & 3 & 4 & 5 & 6 & 7 & 8 \\
\hline Exercices & & 10 & 3 & 2 & 2 & 7 & 1 & 3 & 3 & 2 \\
\hline Mélodies & & 0 & 0 & 0 & 0 & 4 & 0 & 3 & 6 & 2 \\
\hline Pièces & & 0 & 0 & 0 & 0 & 0 & 0 & 0 & 0 & 0 \\
\hline Rythmes utilisés & & 0 & 0 & $\mathrm{a}$ & a & $\begin{array}{c}\mathrm{a}, \mathrm{b}, \mathrm{b} \\
\mathrm{b}, \mathrm{c}\end{array}$ & 0 & $a b c$ & $\mathrm{a}, \mathrm{b}, \mathrm{bb}, \mathrm{c}$ & $\mathrm{a}, \mathrm{b}, \mathrm{bb}, \mathrm{c}$ \\
\hline Explications & & 0,02 & 1 & 2 & 3 & 4 & 5 & 6 & 7 & 8 \\
\hline Images & & 0,01 & 0 & 2 & 3 & 4 & 5 & 6 & 7 & 8 \\
\hline Avec lecture notes & & 0 & 0 & 2 & 3 & 4 & 0 & 6 & 7 & 8 \\
\hline Sans notes & & 0,04 & 1 & 2 & 3 & 4 & 5 & 6 & 7 & 8 \\
\hline Sans explication & & 0 & 0 & 0 & 0 & 0 & 0 & 0 & 0 & 0 \\
\hline Accompagnement/Du & & 0 & 0 & 0 & 0 & 4 & 5 & 6 & 7 & 8 \\
\hline Avec CD & & 0 & 0 & 0 & 0 & 0 & 0 & 0 & 0 & 0 \\
\hline POSTURE & Corps & 0,02 & 0 & 0 & 0 & 0 & 0 & 0 & 0 & 0 \\
\hline POSTURE & Accessoires & 0,02 & 0 & 0 & 0 & 0 & 0 & 0 & 0 & 0 \\
\hline MAIN DROITE & Stabilité & 0 & 0 & 0 & 0 & 0 & 0 & 0 & 0 & 0 \\
\hline MAIN DROITE & Détente & 0 & 0 & 0 & 0 & 0 & 0 & 0 & 0 & 0 \\
\hline \begin{tabular}{|l|l|} 
MAIN DROITE \\
\end{tabular} & Position bras & 0,03 & 0 & 0 & 0 & 0 & 0 & 0 & 0 & 0 \\
\hline MAIN DROITE & Position main & 0,03 & 0 & 0 & 0 & 0 & 0 & 0 & 0 & 0 \\
\hline MAIN DROITE & Position doigts & 0 & 1 & 0 & 0 & 0 & 0 & 0 & 0 & 0 \\
\hline MAIN DROITE & Ongle & 0 & 0 & 0 & 0 & 0 & 0 & 0 & 0 & 0 \\
\hline \begin{tabular}{|l|l} 
MAIN DROITE \\
\end{tabular} & Buté & 0 & 1 & 0 & 0 & 0 & 0 & 0 & 0 & 0 \\
\hline MAIN DROITE & Tiré & 0 & 0 & 0 & 0 & 0 & 0 & 0 & 0 & 0 \\
\hline MAIN DROITE & $\mathrm{i}$ & 0 & 1 & 0 & 0 & 0 & 0 & 0 & 0 & 0 \\
\hline MAIN DROITE & $\mathrm{m}$ & 0 & 1 & 0 & 0 & 0 & 0 & 0 & 0 & 0 \\
\hline MAIN DROITE & $a$ & 0 & 0 & 0 & 0 & 0 & 0 & 0 & 0 & 0 \\
\hline MAIN DROITE & p & 0 & 0 & 0 & 0 & 0 & 0 & 0 & 0 & 0 \\
\hline MAIN DROITE & P appuyé & 0 & 0 & 0 & 0 & 0 & 0 & 0 & 0 & 0 \\
\hline MAIN DROITE & im-mi & 0 & 1 & 0 & 0 & 4 & 0 & 0 & 0 & 0 \\
\hline MAIN DROITE & ia-ai & 0 & 0 & 0 & 0 & 0 & 0 & 0 & 0 & 0 \\
\hline MAIN DROITE & ma-am & 0 & 0 & 0 & 0 & 0 & 0 & 0 & 0 & 0 \\
\hline
\end{tabular}

\section{RÉSUMÉ}

Cet article présente une analyse de méthodes de guitare classique pour débutants puisées dans les programmes d'études pour l'enseignement privé reconnu par le gouvernement du Québec. Pour identifier les principes didactiques sous-jacents à la conception des ouvrages, nous avons analysé les éléments d'apprentissages proposés, leur ordre de présentation ainsi que le type et la quantité d'activités pour chacun. L'apprentissage des notes, de leur lecture et des techniques de jeu dans le but de reproduire le texte musical à l'instrument constitue l'essentiel de ce qui est présenté dans ces méthodes, dans des proportions variables souvent en lien avec l'âge ciblé.

\section{ABSTRACT}

This essay presents an analytical study of classic guitar methods for beginners used in various private school study programs approved by the government of 
Quebec. In order to identify the didactic principles underlying these methods, the elements to be learned, their order, as well as the nature and number of exercises devoted to each of them, have been analyzed. The learning of notes, their reading, the instrumental techniques, aimed at the reproduction of a musical text, are the main aspects on which these methods are focused, in various proportions according to the age of students.

\section{BIOGR APHIE}

Isabelle Héroux est professeure agrégée au département de musique de l'UQAM. Ses intérêts de recherche portent sur tant sur le développement de matériel pédagogique, la didactique instrumentale que la recherche création en interprétation de la guitare et sur les processus créatifs. Isabelle Héroux est membre du laboratoire de recherche en enseignement de la musique du département de musique de l'UQAM (LAREM), du Groupe de recherche interdisciplinaire sur les arts vivants (GRIAV), du Laboratoire de recherche en formation auditive et en didactique instrumentale (LaRFADI) ainsi que de l'Observatoire interdisciplinaire de création et de recherche en musique (OICRM). 